\title{
Triptolide induces Sertoli cell apoptosis in mice via ROS/JNK-dependent activation of the mitochondrial pathway and inhibition of Nrf2-mediated antioxidant response
}

\author{
Yu WANG ${ }^{1, \#}$, Su-han GUO ${ }^{1, \#}$, Xue-jun SHANG ${ }^{2}$, Li-sha $\mathrm{YU}^{1}$, Jian-wei ZHU ${ }^{1}$, Ang ZHAO ${ }^{1}$, Yan-fen ZHOU ${ }^{1}$, Guo-hua $A N^{3}$, \\ Qi ZHANG ${ }^{1, *}$, Bo MA ${ }^{1, *}$ \\ ${ }^{1}$ School of Pharmaceutical Sciences, Nanjing Tech University, Nanjing 210009, China; ${ }^{2}$ Department of Andrology, Jinling Hospital, \\ School of Medicine, Nanjing University, Nanjing 210002, China; ${ }^{3}$ Division of Pharmaceutics and Translational Therapeutics, College of \\ Pharmacy, University of lowa, lowa City, lowa, USA
}

\begin{abstract}
Triptolide (TP), an oxygenated diterpene, has a variety of beneficial pharmacodynamic activities but its clinical applications are restricted due to severe testicular injury. This study aimed to delineate the molecular mechanisms of TP-induced testicular injury in vitro and in vivo. TP (5-50000 nmol/L) dose-dependently decreased the viability of TM4 Sertoli cells with an IC 50 value of 669.5$269.45 \mathrm{nmol} / \mathrm{L}$ at $24 \mathrm{~h}$. TP $(125,250$, and $500 \mathrm{nmol} / \mathrm{L})$ dose-dependently increased the accumulation of ROS, the phosphorylation of JNK, mitochondrial dysfunction and activation of the intrinsic apoptosis pathway in TM4 cells. These processes were attenuated by co-treatment with the antioxidant $\mathrm{N}$-acetyl cysteine (NAC, $1 \mathrm{mmol} / \mathrm{L})$. Furthermore, TP treatment inhibited the translocation of Nrf2 from cytoplasm into the nucleus as well as the expression of downstream genes NAD(P)H quinone oxidoreductase1 (NQ01), catalase (CAT) and hemeoxygenase 1 (HO-1), thus abrogating Nrf2-mediated defense mechanisms against oxidative stress. Moreover, siRNA knockdown of Nrf2 significantly potentiated TP-induced apoptosis of TM4 cells. The above results from in vitro experiments were further validated in male mice after oral administration of TP $\left(30,60\right.$, and $120 \mathrm{mg} \cdot \mathrm{kg}^{-1} \cdot \mathrm{d}^{-1}$, for $\left.14 \mathrm{~d}\right)$, as evidenced by the detected indexes, including dose-dependently decreased SDH activity, increased MDA concentration, altered testicle histomorphology, elevated caspase-3 activation, apoptosis induction, increased phosphorylation of JNK, and decreased gene expression of NQO1, CAT and HO-1 as well as nuclear protein expression of Nrf2 in testicular tissue. Our results demonstrate that TP activates apoptosis of Sertoli cells and injury of the testis via the ROS/JNK-mediated mitochondrial-dependent apoptosis pathway and down-regulates Nrf2 activation.
\end{abstract}

Keywords: triptolide; Sertoli cells; MAPKs; apoptosis; ROS; Nrf2; NAC

Acta Pharmacologica Sinica (2018) 39: 311-327; doi: 10.1038/aps.2017.95; published online 14 Sep 2017

\section{Introduction}

Tripterygium wilfordii multiglycoside (GTW), a traditional Chinese herbal medicine, has long been used to treat inflammatory and immune diseases, including lupus erythematosus, rheumatism arthritis and pouchitis ${ }^{[1,2]}$. With the development of technology for the modernization of Traditional Chinese Medicine (TCM), Triptolide (TP, $\mathrm{C}_{20} \mathrm{H}_{24} \mathrm{O}_{6}$, Figure 1A), a diterpene epoxide extracted from the roots

\footnotetext{
"These authors contributed equally to this work.

* $T o$ whom correspondence should be addressed.

E-mail mabo201012@njtech.edu.cn (Bo MA); nancyzhang03@hotmail.com (Qi ZHANG)

Received 2017-01-15 Accepted 2017-06-19
}

of GTW, has been recognized as a major active ingredient responsible for the pharmacological and toxic effects of $\mathrm{GTW}^{[3-5]}$. On the one hand, TP has strong and beneficial pharmacodynamic activities, such as anti-rheumatic, antiinflammatory, anti-microbial and anti-tumor activities ${ }^{[6-10]}$; on the other hand, it also exerts a narrow therapeutic window and severe adverse effects on the liver, kidney, heart, and reproductive system and on hematopoiesis. Among those side effects of TP, reproductive toxicity is the most significant, owing to the high incidence rate. Therefore, TP has been used as a model drug in an animal male infertility disease model to investigate the pathological mechanism of male infertility and related drug effects. Furthermore, illuminating the underlying mechanism of inducing toxicity would aid in alleviating ${ }^{[11-13]}$ or 
A

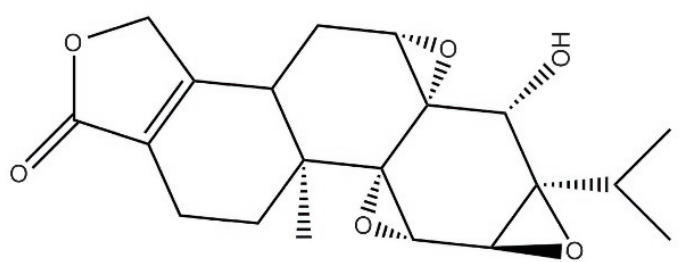

C

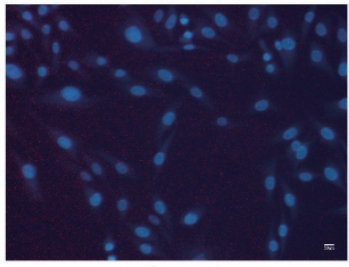

Control

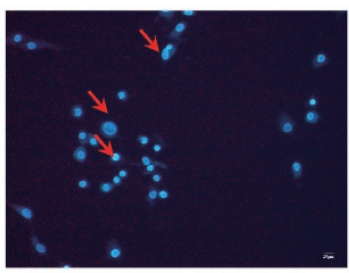

$250 \mathrm{nmol} / \mathrm{L}$

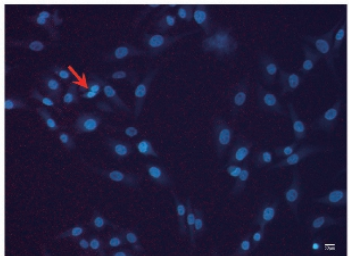

$125 \mathrm{nmol} / \mathrm{L}$

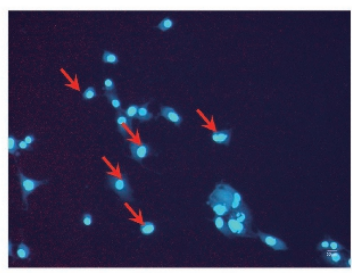

$500 \mathrm{nmol} / \mathrm{L}$

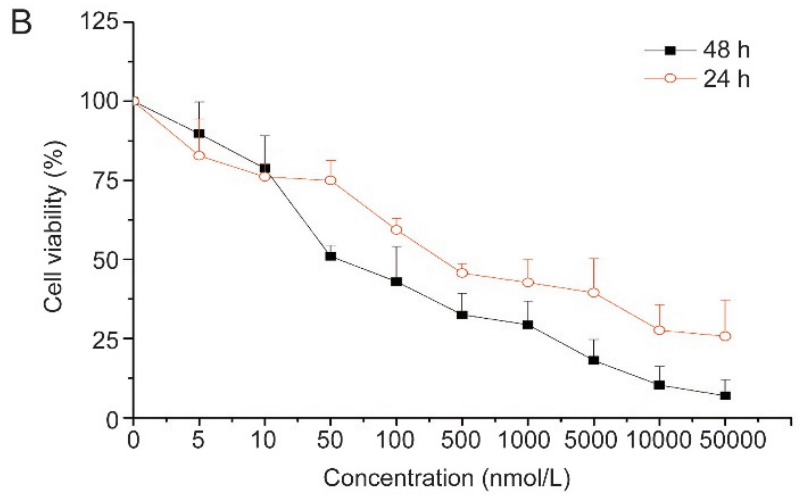

$\mathrm{E}$

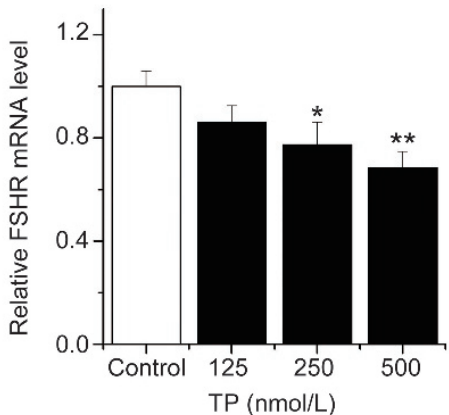

D

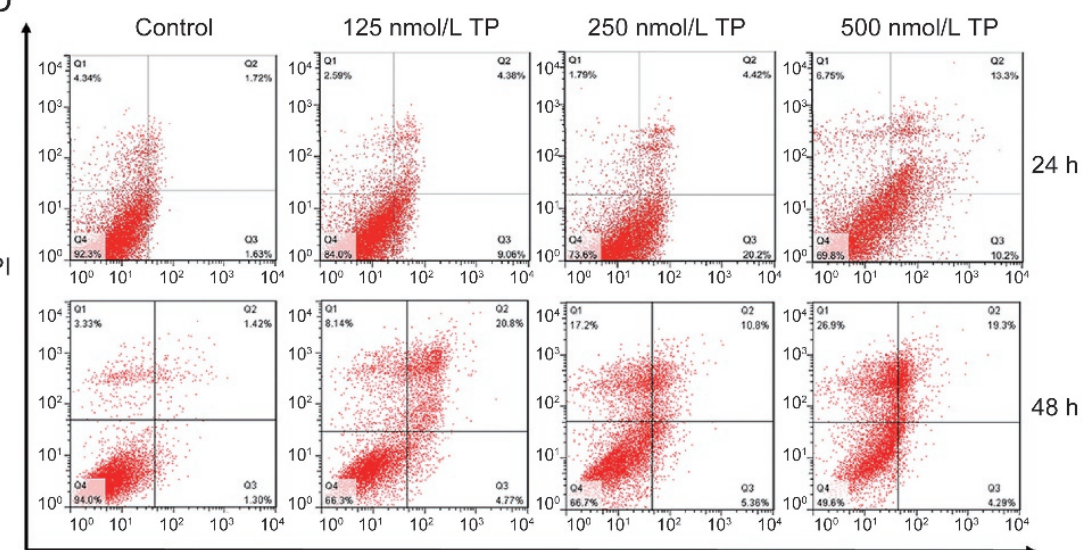

Annexin V-FITC

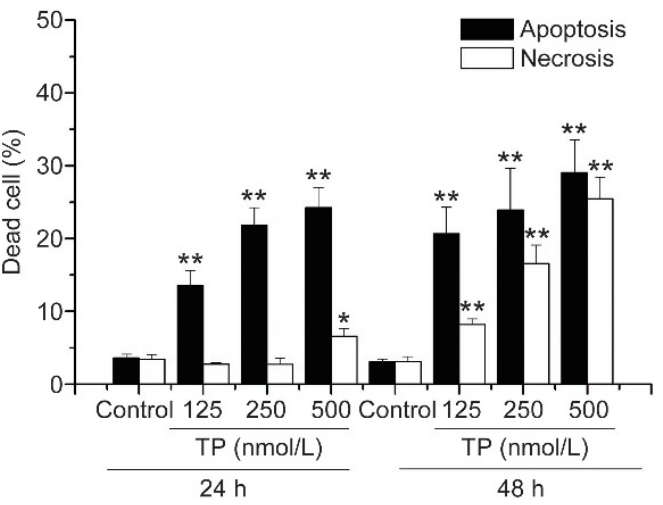

Figure 1. Effects of TP on cell viability and apoptosis in the TM4 Sertoli cells. (A) Chemical structure of TP. (B) Viability of cells with various concentrations of TP $(5-10000 \mathrm{nmol} / \mathrm{L})$ for 24 and $48 \mathrm{~h}$ was determined by MTT assay, respectively. (C) Apoptotic morphological changes were evaluated by fluorescent microscopy using Hoechst 33258 staining. Arrows indicate chromatin condensation and DNA fragmentation. (D) Apoptosis in TM4 cells was detected after 24 and $48 \mathrm{~h}$ of treatment with 125, 250 and $500 \mathrm{nmol} / \mathrm{L}$ TP by Annexin V-FITC/PI binding and measured by flow cytometry analysis. The chart illustrates apoptosis proportion from three separate experiments. (E) The mRNA expression of FSHR was detected by Real time PCR in TM4 cells. Data represent the mean \pm SD of three independent experiments. ${ }^{*} P<0.05,{ }^{* *} P<0.01$ vs untreated control group.

avoiding adverse reactions via combination drug therapy ${ }^{[14]}$.

TP has been reported to have a wide variety of adverse effects in the male reproductive system, including decreasing the diameter of seminiferous tubules and the number of various spermatogenic cells in male rats ${ }^{[15]}$, decreasing the weights of the testis and epididymis ${ }^{[16]}$ and disrupting sperm morphology ${ }^{[17]}$. Testis tissue is a main site of toxicity because TP can easily permeate into the blood-testis barrier after administration and increase exposure to TP in testicular tissue $^{[18]}$. Our previous studies conducted in TP-treated mice have shown clear testicular injury, including an abrogated microstructure, peritubular cells, developing germ cells, as well as changes in the gonad index of the testis and the energy metabolism system ${ }^{[19]}$. Testicular tissues are mainly composed of two types of cells: Sertoli cells (SCs) and Leydig cells. Leydig cells are the testosterone-producing cells of the testis, and SCs in the seminiferous tubule of the testis play a pivotal role in supporting the formation of the testis and blood-testis barrier, thus protecting germ cells from harmful influences ${ }^{[20]}$. SCs act as nurse cells and manage the processes 
of spermatogenesis by providing nutrition and an adaptive environment for germ cell survival and development during mammalian spermatogenesis. Therefore, impairment of SCs may have negative effects on spermatogenesis, sperm production and morphological changes in the testis.

Some proposed molecular mechanisms of TP toxicity include the induction of apoptosis ${ }^{[21]}$, oxidative stress ${ }^{[22]}$, disruption of the cAMP/PKA pathway ${ }^{[23]}$, mitochondrial injury ${ }^{[24]}$, and decreased tight junctions ${ }^{[25]}$. However, little information has been reported concerning the toxic effects of TP on SCs, and the precise molecular mechanism underlying its toxicity remains unclear. As previously reported, TP can cause tissue injury via oxidative stress ${ }^{[26]}$. Increased oxidative stress in testicular tissue may be a potential mechanism of TPinduced testis tissue injury, reproductive system disorders and male sterility. The reactive oxygen species (ROS) generated can decrease the mitochondrial membrane potential $(\Delta \psi \mathrm{m})$, induce mitochondrial dysfunction, and eventually lead to apoptosis. It is currently believed that the induction of apoptosis and the production of ROS are essential steps in the testicular injury triggered by TP. All these actions involve a complex array of intracellular signaling pathways including the mitogen-activated protein kinase (MAPK) pathways, caspase-3 pathways, mitochondrial pathways and nuclear factor erythroid 2-related factor 2 (Nrf2) pathways.

MAPKs regulate diverse processes including cell proliferation, differentiation, apoptosis, gene expression, protein synthesis and secretion. In mammals, at least 3 families of MAPKs have been identified: extracellular signal-regulated kinase (ERK) 1/2, c-Jun N-terminal kinase (JNK), and p38. Recent studies have shown that activation of MAPK signaling pathways plays important roles in the development of $\mathrm{SCs}^{[27,28]}$. The activation of JNKs is principally associated with SC exposure to reproductive toxicants ${ }^{[29-31]}$. It is highly probable that ROS accumulation, the mitochondrial pathway, and the JNK signaling cascade are involved in TP-induced apoptosis of SCs, but this possibility has not yet been validated by reported evidence.

The Nrf2 system is known as one of the most important defense mechanisms against oxidative stress and electrophilic insults. Nrf2 is a master transcription factor in the response to oxidative stress that binds to antioxidant responsive elements (AREs) and induces the expression of several protective enzyme including $\mathrm{NAD}(\mathrm{P}) \mathrm{H}$ quinone oxidoreductase1 (NQO1), catalase (CAT), glutamate-cysteine ligase (GCL), hemeoxygenase-1(HO-1), and glutathione $S$-transferase(GST). It controls cell proliferation and differentiation via the regulation of ROS. Expression of a functional Nrf2 system in the male reproductive tract (testis and epididymis) may play a potentially protective role against oxidative stress damage ${ }^{[32,33]}$.

Therefore, in the present work, we aimed to delineate the molecular mechanism of testicular injury caused by TP both in vivo and in vitro. TM4 cells, representing a mouse SC model, were used to explore whether exposure of SCs to TP leads to potential cytotoxicity and induces apoptosis. The underlying mechanisms were then elucidated by investigating the involvement of possible apoptosis-related hallmarks including the generation of ROS, changes in mitochondrial membrane potential, and levels of cytochrome $c$, caspase-3, MAPKs and Nrf2 in vitro. ROS scavenger $N$-acetyl-cysteine (NAC) and the specific JNK inhibitor (SP600125) were evaluated in particular to verify the underlying mechanisms of TP-induced apoptosis. The mechanisms were further validated through experiments including testis histopathologic analysis, TUNEL assays and immunohistochemical detection in vivo. To our knowledge, these are the first results demonstrating the cytotoxic effects and underlying molecular mechanisms of TP-induced apoptosis in SCs.

\section{Materials and methods Reagents}

Cell culture medium, DMEM/F12, ampicillin and streptomycin were purchased from Gibco BRL (Grand Island, NY, USA). Fetal bovine serum and donor equine serum were obtained from HyClone (South Logan, Utah, USA). TP was purchased from $\mathrm{Xi}^{\prime}$ an Kai Lai Biological Engineering ( $\mathrm{Xi}^{\prime}$ an, China). Dimethyl sulfoxide (DMSO), N-acetyl-L-cysteine (NAC), DAPI, 3-(4, 5-dimethylthiazol-2-yl)-2, 5-diphenyl tetrazolium bromide (MTT) and 6-carboxy-2', $7^{\prime}$-dichlorofluorescein diacetate (DCFDA) were purchased from Sigma-Aldrich (St Louis, MO, USA). SB203580, PD98059 and SP600125 were obtained from Calbiochem (San Diego, CA, USA). Primary antibodies to $\beta$-Actin, ERK1/2, p-ERK1/2, p-P38, P38, p-JNK, JNK, cleaved caspase- 3 and PARP were purchased from Cell Signaling Technology (SCT, MA, USA), and Bax, Bcl-2 and cytochrome $c$ were obtained from Bioworld Technology (Bioworld Technology, USA).

\section{Cell culture}

The TM4 SCs were purchased from the American Tissue Culture Collection. DMEM/F12 enriched with $2.5 \%$ fetal bovine serum and $5 \%$ donor equine serum containing $1 \%$ penicillin-streptomycin solution was used for cell culture. All cells were maintained at $37^{\circ} \mathrm{C}$ in a humidified atmosphere containing $5 \% \mathrm{CO}_{2}$.

\section{Cell viability assay}

TP was dissolved in DMSO at a concentration of $100 \mathrm{mmol} / \mathrm{L}$ as a stock solution and stored at $-20{ }^{\circ} \mathrm{C}$ until use. To evaluate the toxic effect of TP on cell growth, TM4 cells were seeded at a density of $5 \times 10^{3}$ cells/well in 96-well culture plates for $48 \mathrm{~h}$. The medium was then discarded and replaced with $150 \mu \mathrm{L}$ of fresh serum-free medium containing various concentrations (5-10000 nmol/L) of TP and cultured for $24 \mathrm{~h}$ and $48 \mathrm{~h}$, respectively. After treatment, cell viability was determined by the MTT assay. Briefly, $15 \mu \mathrm{L}$ of MTT stock solution $(5 \mathrm{mg} / \mathrm{mL}$ ) was added to each well and incubated for $4 \mathrm{~h}$ at $37^{\circ} \mathrm{C}$ in the dark. The culture medium was then removed, and $100 \mu \mathrm{L}$ DMSO was added to dissolve the formazan crystals. The absorbance was then measured at $570 \mathrm{~nm}$.

\section{Cellular morphology of TM4 cells, DNA electrophoresis analyses and Hoechst 33258 staining}

The TM4 $\left(2 \times 10^{4}\right)$ cells were seeded on 24 -well microplates for 
$24 \mathrm{~h}$ of growth. Photographs were obtained at $24 \mathrm{~h}$ after drug intervention by using a Nikon Inverted Microscope (Nikon TS100, Nikon, Tokyo, Japan). In addition, DNA samples isolated from normal and TP-treated cells and apoptosis were determined by evaluating DNA fragmentation by agarose gel electrophoresis. The cells were fixed for $10 \mathrm{~min}$ in $4 \%$ paraformaldehyde (PFA) and then stained with Hoechst 33258 (5 $\mu \mathrm{g} / \mathrm{mL}$ ) for $5 \mathrm{~min}$ in the dark. The cells were observed under a fluorescence microscope.

\section{Annexin V-FITC/propidium iodide (PI) staining}

Cell apoptosis was quantified with an Annexin V-FITC/PI apoptosis kit (Beyotime Institute of Biotechnology, Nantong, Jiangsu, China). The TM4 $\left(5 \times 10^{5} / \mathrm{mL}\right)$ cells were seeded on 6-well microplates and grown for $48 \mathrm{~h}$. After treatment with TP $(125,250,500 \mathrm{nmol} / \mathrm{L})$ for $24 \mathrm{~h}$ and $48 \mathrm{~h}$, TM4 cells were digested with trypsin lacking EDTA, centrifuged at $1000 \mathrm{r} / \mathrm{min}$ for $5 \mathrm{~min}$ at $4{ }^{\circ} \mathrm{C}$, washed twice with cold PBS, and re-suspended in assay buffer at a concentration of $1 \times 10^{6}$ cells $/ \mathrm{mL}$. Then, the collected TM4 cells were incubated in buffer containing $1 \mu \mathrm{g} / \mathrm{mL}$ PI and $5 \mu \mathrm{L}$ Annexin V-FITC in the dark at room temperature for $15 \mathrm{~min}$, followed by examination using a FACScan flow cytometer (Becton-Dickson, Immunocytometry System, San Jose, CA, USA). FITC and PI fluorescence were measured by excitation with a $488 \mathrm{~nm}$ argon laser. Finally, a dot plot of PI fluorescence (y-axis) versus FITC fluorescence (x-axis) was prepared.

\section{Measurement of the mitochondrial membrane potential $(\Delta \psi \mathrm{m})$}

The mitochondrial membrane potential $(\Delta \psi \mathrm{m})$ was assayed by JC-1 staining and flow cytometry. In brief, TM4 cells were treated with TP $(125,250,500 \mathrm{nmol} / \mathrm{L})$ or left untreated for $24 \mathrm{~h}$ and $48 \mathrm{~h}$, and then the cells were harvested, washed and incubated with JC-1 $(10 \mu \mathrm{g} / \mathrm{mL})$ for $30 \mathrm{~min}$ at $37^{\circ} \mathrm{C}$. The level of $\Delta \psi \mathrm{m}$ was analyzed by flow cytometry.

\section{Detection of intracellular ROS}

Production of ROS was observed in TM4 cells by fluorescence microscopy using DCFDA as the molecular probe. In brief, TM4 cells were treated with or TP $(125,250,500 \mathrm{nmol} / \mathrm{L})$ or left untreated for $6 \mathrm{~h}$, and then the cells $\left(2 \times 10^{5}\right)$ were loaded with $10 \mu \mathrm{mol} / \mathrm{L}$ DCFDA for $30 \mathrm{~min}$ at $37^{\circ} \mathrm{C}$ in the dark, washed twice with PBS, fixed with $4 \%$ paraformaldehyde, and washed with PBS. Stained cells were observed under a fluorescence microscope, and cells were also collected, and their fluorescence intensities assessed using a Bio-Tek Fluorescence microplate reader at an excitation wavelength of $480 \mathrm{~nm}$ and an emission wavelength of $530 \mathrm{~nm}$.

\section{Measurement of lipid peroxidation and glutathione}

Intracellular glutathione (GSH) content and MDA levels in cell culture medium were determined using a colorimetric microplate assay (Nanjing Jiancheng Bioengineering Institute, Nanjing, Jiangsu, China). In brief, TM4 cells were treated with TP $(125,250$, and $500 \mathrm{nmol} / \mathrm{L})$ or left untreated for $12 \mathrm{~h}$, and culture supernatants were collected by centrifugation at 3500 $\mathrm{r} / \mathrm{min}$ for $10 \mathrm{~min}$ at $4{ }^{\circ} \mathrm{C}$. The MDA levels in the supernatant were used for the assays, according to the manufacturer's instructions. TP-treated cells were detached using $0.25 \%$ trypsin-1 mmol/L EDTA and harvested via centrifugation at 1000 $\mathrm{r} / \mathrm{min}$ for $5 \mathrm{~min}$ at $4{ }^{\circ} \mathrm{C}$. The cell samples were then prepared and intracellular GSH contents detected, according to the manufacturer's protocols. MDA and GSH content was normalized to the total protein concentration using the standard method supplied with the Thermo Scientific Pierce BCA Protein assay (Thermo, Rockford, USA).

\section{Western blot analysis}

TM4 cells were harvested and lysed for $30 \mathrm{~min}$ in cell lysis buffer with protein extraction reagent containing $1 \mathrm{mmol} / \mathrm{L}$ phenylmethylsulfonylfluoride (PMSF), complete EDTA-free protease inhibitor cocktail (Roche Diagnostics, Indianapolis, IN) and $5 \mathrm{mmol} / \mathrm{L}$ sodium orthovanadate (a phosphatase inhibitor), according to the instructions provided with the Cyto-plasmic Protein Extraction Kit, Nucleoprotein Extraction Kit and Cell Mitochondria Isolation Kit (Beyotime Institute of Biotechnology, Nantong, Jiangsu, China). Equal amounts of protein $(20 \mu \mathrm{g} /$ lane $)$ were subjected to sodium dodecyl sulfate-polyacrylamide gel electrophoresis (SDS-PAGE). Subsequently, Western blotting was performed at $80 \mathrm{~V}$ for $30 \mathrm{~min}$ and $120 \mathrm{~V}$ for $65 \mathrm{~min}$, and then the gel was transferred to polyvinylidene difluoride (PVDF) membranes (Bio-Rad, Hercules, CA, USA). The membranes were blocked with $5 \%$ BSA solution for $2 \mathrm{~h}$ at room temperature. The membranes were incubated overnight with a polyclonal primary antibody and TBS, $5 \%$ BSA and $0.1 \%$ Tween-20. Primary antibodies to the following proteins were used: $\beta$-actin, ERK1/2, p-ERK1/2, p-p38, p38, p-JNK, JNK, cytochrome $c$, Nrf2, cleaved caspase-3 and PARP (1:1000 in PBS, Cell Signaling Technology, USA); Bax and Bcl-2 (1:750 in PBS, Bioworld Technology, USA). After being washed four times with Tris-buffered saline containing $0.1 \%$ Tween-20, the membranes were incubated with secondary HRP-conjugated anti-rabbit IgG antibody (1:1000 Cell Signaling Technology, USA) for $2 \mathrm{~h}$ at room temperature. Finally, the protein bands were visualized with an ECL detection system (Millipore, Billerica, MA, USA). Data were normalized to the corresponding internal reference $\beta$-actin or ERK1/2 (p38 or JNK). The immunoblotting densitometry analysis was performed by using Clinx Chemi Image Analysis software (Clinx Science, Shanghai, China). All samples were analyzed in triplicate.

\section{Immunofluorescence analysis of $\mathrm{Nrf2}$ expression}

To localize Nrf2 expression in cells, immunofluorescence was performed. After treatment with TP for $3 \mathrm{~h}$, cells were harvested, washed in ice-cold PBS, fixed with $4 \%$ paraformaldehyde in PBS, pH 7.4, for $15 \mathrm{~min}$ at $4{ }^{\circ} \mathrm{C}$ and then permeated with $0.1 \%$ Triton $X-100$. Fixed cells were washed with ice-cold PBS and incubated with anti-Nrf2 antibody (1:200 in PBS) for $12 \mathrm{~h}$ at $4{ }^{\circ} \mathrm{C}$. After the washes, the slides were incubated for $1 \mathrm{~h}$ at $37^{\circ} \mathrm{C}$ with DyLight 488 -conjugated Affini-Pure goat anti-rabbit IgG secondary antibodies. The nuclei were then 
stained with DAPI solution. DAPI and Nrf2 fluorescence was then evaluated by confocal laser scanning microscopy (CLSM), and confocal images were obtained using a Zeiss confocal laser microscope (ZEISS LSM 700) and analyzed using MetaFluorV6.3 (Universal Imaging, Downingtown, PA, USA).

\section{Reverse transcription-PCR}

Total RNA was isolated from the cells with TRIzol reagent according to the manufacturer's guidelines (TRIzol, TaKaRa Biotechnology, Dalian, China), after TP (125, 250 and 500 $\mathrm{nmol} / \mathrm{L})$ treatment for $6 \mathrm{~h}$. The concentration and purity of the RNA were quantified by measuring the absorbance at 260 $\mathrm{nm}$ and $280 \mathrm{~nm}$ (NanoDrop One, Thermo, USA). The primer sequences (Supplemental material Table S1) for NQO1, HO-1, GSTA-1, Gclc, FSHR (treatment for $2 \mathrm{~h}$ ) and GAPDH were synthesized by Invitrogen China Inc (Shanghai, China). Reverse transcription was performed using an All-In-One RT MasterMix Kit (Applied Biological Materials Inc, Canada) according to the manufacturer's instructions. Real-time quantitative PCR was performed using a CFX96 Touch Real-Time PCR detection system (Bio-Rad, USA). Each PCR assay was carried out in a single well of a 96-well optical reaction plate with EvaGreen 2× qPCR MasterMix (Applied Biological Materials Inc, Canada) in accordance with the manufacturer's instructions. The relative gene expression levels were calculated by using the comparative threshold cycle $(\mathrm{Ct})$ method $\left(2^{-\Delta \Delta \mathrm{Ct}}\right)$ by normalizing the target mRNA $\mathrm{Ct}$ values to those of GAPDH. All samples were analyzed in triplicate.

\section{siRNA transient transfection}

Nrf2 was silenced in TM4 cells by using Nrf2 siRNA. The following siRNA-encoding DNA oligonucleotides containing inner palindromic sequences (sense: 5'-CGAGAAGUGUUUGACUUUATT-3'; antisense: 5'-UAAAGUCAAACACUUCUCGTT-3', and scramble siRNA (sense: 5' -UUCUCCGAACGUGUCACGUTT-3'; antisense: 5'-ACGUGACACGUUCGGAGAATT-3') were synthesized (GenePharma, USA). TM4 cells were transiently transfected with Nrf2 siRNA or scrambled siRNA as a negative control by using Lipofectamine 2000 (Invitrogen, Carlsbad, CA, USA) according to the associated protocol. Moreover, at $24 \mathrm{~h}$ post-transfection, the cells were collected for Western blot analysis or treated with TP.

\section{Animals and in vivo research}

Thirty-two male ICR mice weighing approximately 18-22 g were obtained from the Animal Center of Nanjing Medical University (NJMU, Nanjing, China) and randomly assigned to four groups $(n=8)$. In the control group, mice received the following by gavage once daily every day for 14 days: normal solvent for TP (TP dissolved in ethanol/0.9\% saline $=1: 9$, vol); TP (low, medium and high dose): TP at doses of 30, 60 and $120 \mu \mathrm{g} / \mathrm{kg}$ po, respectively. All mice were maintained in an environmentally controlled room at a temperature of $22 \pm 2{ }^{\circ} \mathrm{C}$, with a relative humidity of $50 \% \pm 10 \%$ and an approximately 12/12-h light/dark cycle. They were given free access to food and water until $12 \mathrm{~h}$ before sacrifice. Testicular tissues were removed and weighed to calculate the testis index (testis weight/body weight) after treatment for 14 days. All animal experiments were performed according to the Guidelines for the Animal Ethics Committee of Nanjing Tech University (Nanjing, China).

Succinate dehydrogenase, malondialdehyde, and activation of caspase-3 in testicular tissue

Succinate dehydrogenase (SDH) in the mitochondrial membrane is a key mitochondrial enzyme. The biochemical indicator of malondialdehyde (MDA) indicates the formation of lipid peroxide. The SDH and SOD activities, as well as MDA levels in testicular tissue, were detected by using classical colorimetric methods with commercial kits (Nanjing Jiancheng Bioengineering Institute, Nanjing, Jiangsu, China), according to the manufacturer's instructions. The results are expressed as units/mg of protein. Activation of caspase-3 in testicular tissue was detected by using a caspase- 3 colorimetric assay kit (Beyotime Institute of Biotechnology, Nantong, Jiangsu, China) according to the manufacturer's instructions.

\section{Histopathologic analysis of the testis}

A histopathologic study was performed at the end of the experiment. As described in a previous report ${ }^{[19]}$, the right sides of the mouse testis were removed at the end of the experiment, cut in the middle and fixed with $10 \%$ buffered formalin solution ( $\mathrm{pH} 7.4$ ) at $4{ }^{\circ} \mathrm{C}$ for 2 weeks. The fixed samples were then dehydrated through a graded alcohol series, embedded in paraffin and sliced. After staining with hematoxylin and eosin (H \& E), the pathological changes in the testicular tissues were observed under a light microscope (Nikon ECLIPSE 80i, Nikon, Tokyo, Japan).

\section{TUNEL assay}

The terminal DNA transferase-mediated dUTP nick end labeling (TUNEL) assay was used to determine apoptotic DNA breaks. Paraffin-embedded testis tissue sections were used to assess TP-induced apoptosis. TUNEL labeling was performed with an in situ cell apoptosis detection kit for the detection of paraffin sections (KeyGEN BioTECH, Nanjing, China), according to the manufacturer's recommendations. The slides were observed under an optical microscope.

\section{Immunohistochemistry}

Formalin-fixed paraffin-embedded testicular tissue sections from animals treated with TP or left untreated were deparaffinized and soaked in graded concentrations of ethyl alcohol. The sections were then soaked in sodium citrate buffer $(\mathrm{pH}$ 6.0) and heated in a microwave oven for antigen retrieval. After being washed with PBS, the slides were blocked with $10 \%$ horse serum for $1 \mathrm{~h}$ at room temperature. Moreover, the slides were incubated with primary antibodies against p-JNK (1:50) overnight at $4{ }^{\circ} \mathrm{C}$. After being washed 4 times with $1 \times$ TBST for $5 \mathrm{~min}$ each, the slides were incubated with $100 \mu \mathrm{L}$ per slide of diluted conjugated secondary antibody for $30 \mathrm{~min}$ at room temperature. The slides were then stained with DAB 
and observed under a light microscope (Nikon ECLIPSE 80i, Nikon, Tokyo, Japan).

\section{Statistical analysis}

All values are presented as the mean $\pm \mathrm{SD}$ and analyzed by one-way ANOVA. Statistical significance was determined as $P<0.05$. All figures were obtained from at least three independent experiments.

\section{Results}

\section{TP-induced apoptosis of TM4 cells in vitro}

First, we determined the effect of TP on cell viability. As shown in Figure 1B, TP significantly inhibited cell growth in both a dose-dependent and a time-dependent manner. The $\mathrm{IC}_{50}$ values were $669.5 \pm 269.45 \mathrm{nmol} / \mathrm{L}$ and $118.4 \pm 34.35 \mathrm{nmol} / \mathrm{L}$ for 24 and $48 \mathrm{~h}$, respectively. The cells became shrunken or lost their adherence after being incubated with various concentrations of TP $(50,100,500,1000$, and $5000 \mathrm{nmol} / \mathrm{L})$ for $24 \mathrm{~h}$ (Supplementary materials Figure S1). In addition to the morphological observations, chromatin condensation and DNA fragmentation were observed in TP-treated TM4 cells by Hoechst 33258 staining (Figure 1C). The cytotoxic effect of TP was confirmed by flow cytometry. The early apoptotic cells (Annexin $\mathrm{V}^{+} / \mathrm{PI}^{-}$) fell into the lower right quadrant and the late apoptotic cells (Annexin $\mathrm{V}^{+} / \mathrm{PI}^{+}$) fell into the right upper quadrant. As shown in Figure 1D, the apoptosis rate (dot plot in the lower right quadrant and right upper quadrant) was significantly increased after treatments with TP $(125,250$ and $500 \mathrm{nmol} / \mathrm{L}$ ) for $24 \mathrm{~h}$ and $48 \mathrm{~h}$, respectively, in a concentration-dependent manner. Flow cytometric analysis showed that $500 \mathrm{nmol} / \mathrm{L}$ induced an apoptotic to necrotic cell ratio of up to $30 \%$ for $24 \mathrm{~h}$ (early and late apoptotic and necrotic cells) and $50 \%$ apoptotic cells at $48 \mathrm{~h}$. Thus, we further assessed the DNA fragmentation pattern by agarose gel electrophoresis to investigate TP-induced apoptosis of TM4 cells. The data showed that TP treatment resulted in DNA ladder fragmentation in a concentration-dependent manner (Supplemental material Figure S2). Our results also showed that the gene expression of FSHR was significantly decreased in response to 250 and $500 \mathrm{nmol} / \mathrm{L}$ TP treatment for $2 \mathrm{~h}$ as compared with that in the control group (Figure 1E). These results indicated that TP-induced apoptosis of Sertoli cells and damaged their physiological functions.

\section{Role of ROS generation in TM4 cell apoptosis induced by TP}

To investigate whether ROS play a pivotal role in TP-induced apoptosis, DCFH-DA, a fluorescent probe, was used to detect cellular ROS levels. In our experiment, TP treatment (125, 250 , and $500 \mathrm{nmol} / \mathrm{L}$ ) for $6 \mathrm{~h}$ induced a marked increase in DCFH-DA fluorescence in a concentration-dependent manner, as compared with the fluorescence in the control group, which exhibited only weak green fluorescence. The results are shown in Figure 2A and 2B. To further determine whether the apoptosis was related to the levels of ROS, cells were coincubated with the antioxidant NAC together with TP (500 nmol/L). NAC abrogated the TP-induced decline in TM4 cell viability introduced by TP (Figure $2 \mathrm{~A}$ and $2 \mathrm{~B}$ ) and caused a $64.91 \%$ decrease in the rate of apoptosis in NAC-treated TM4 cells. Data obtained from MTT analysis are shown in Figure 2C. Moreover, TP treatment induced a concentration-dependent decrease in GSH levels (Figure 2D) and a concentrationdependent increase in MDA levels (Figure 2E) in TM4 cells. These findings indicated that TP causes apoptosis of TM4 cells by promoting ROS generation in response to oxidative stress damage.

\section{Mitochondrial-dependent apoptotic signals in TM4 cells induced by TP}

The collapse in mitochondrial membrane potential $\left(\Delta \psi_{\mathrm{m}}\right)$ is a crucial step in triggering activation of the apoptotic cascade. To further investigate whether TP-induced SC apoptosis is regulated via mitochondrial dysfunction, the mitochondrial membrane potential was determined by flow cytometry using JC-1 staining. As shown in Figure 3A, the decrease in $\Delta \psi \mathrm{m}$ in TM4 cells exhibited a dose-dependent sensitivity to TP at $24 \mathrm{~h}$ and $48 \mathrm{~h}$, as indicated by the increased green fluorescence and decreased red fluorescence.

We then measured the changes in Bcl-2 family protein expression to determine whether TP-induced apoptosis by triggering the mitochondrial apoptosis pathway. The Western blot results indicated that Bax protein levels were significantly increased in the 250 or $500 \mathrm{nmol} / \mathrm{L}$ TP groups, whereas antiapoptotic protein (Bcl-2) was significantly attenuated by TP for 24 and $48 \mathrm{~h}$, especially at a high concentration (Figure 3B). As a result, the Bax/Bcl-2 ratios (pro-apoptotic/anti-apoptotic) were enhanced after TP treatment in TM4 cells (Figure 3C).

Cytochrome $c$ (Cyt $c$ ) release was linked to the decrease in $\Delta \psi \mathrm{m}$ and the mitochondrial apoptosis pathway. Cyt $c$ release from mitochondria activates caspase apoptotic pathways, which in turn activate the executioner caspase-3 protein and eventually result in apoptosis. We investigated the possible involvement of the release of Cyt $c$ in the process of TPinduced apoptosis by comparing the protein expression of cytochrome $c$ in the cytoplasm and mitochondria by Western blot analysis. Cells were treated with different concentrations of TP for 24 and $48 \mathrm{~h}$, and the protein expression of cytochrome $c$ in the cytoplasm was found to be significantly upregulated in concentration-dependent and time-dependent manners in TP-treated TM4 cells. Our data indicated that TP promoted the release of $\mathrm{Cyt} c$ from mitochondria into the cytoplasm (Figure 3B).

Caspases, particularly caspase- 3 , are key mediators of cell death. Cleaved caspase-3 was significantly regulated in a concentration-dependent manner in TP-treated TM4 cells. Additionally, together with the activation of caspase-3, full-length PARP $(116 \mathrm{kDa})$ protein was fragmented to yield an $85-\mathrm{kDa}$ fragment. This finding indicated that TP-induced apoptosis in TM4 cells (Figure 3B). Together, the results showed that TP triggers the mitochondrial caspase apoptotic pathway via a series of cascade reactions including an enhanced Bax/ Bcl-2 ratio, a loss of mitochondrial membrane potential, Cyt $c$ release, and the caspase cascade. 


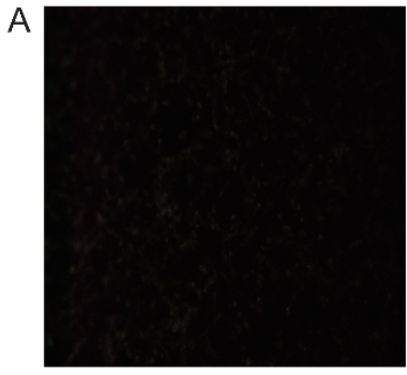

Control

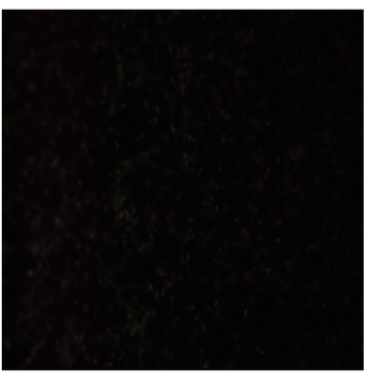

$\mathrm{NAC}(1 \mathrm{mmol} / \mathrm{L})$

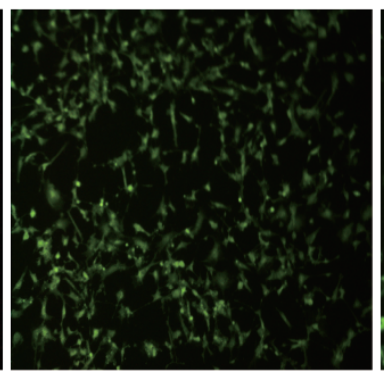

$125 \mathrm{nmol} / \mathrm{L}$

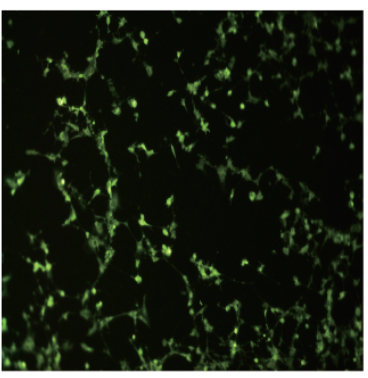

$250 \mathrm{nmol} / \mathrm{L}$

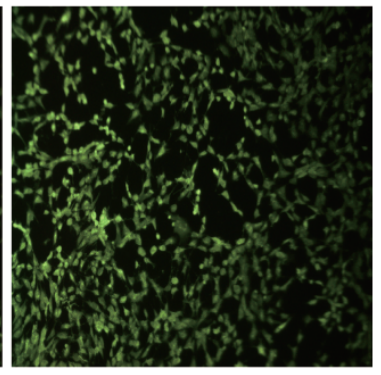

$500 \mathrm{nmol} / \mathrm{L}$

TP

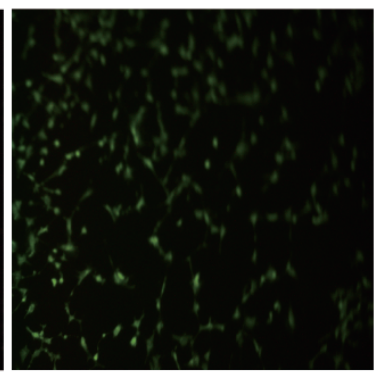

TP $(500 \mathrm{nmol} / \mathrm{L})+\mathrm{NAC}(1 \mathrm{mmol} / \mathrm{L})$

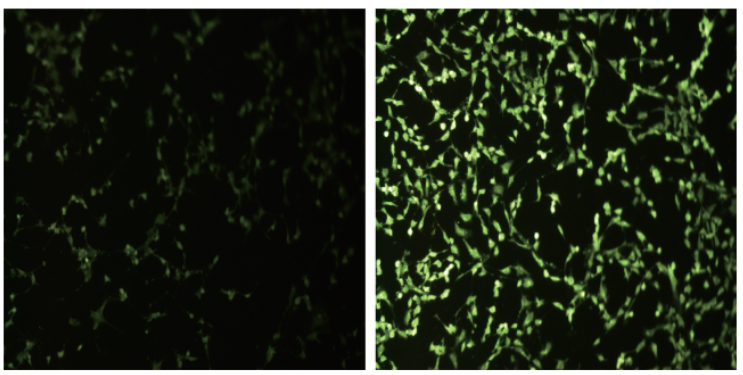

SP600125 $(10 \mu \mathrm{mol} / \mathrm{L}) \quad$ TP $(500 \mathrm{nmol} / \mathrm{L})+\mathrm{SP} 600125(10 \mu \mathrm{mol} / \mathrm{L})$

C
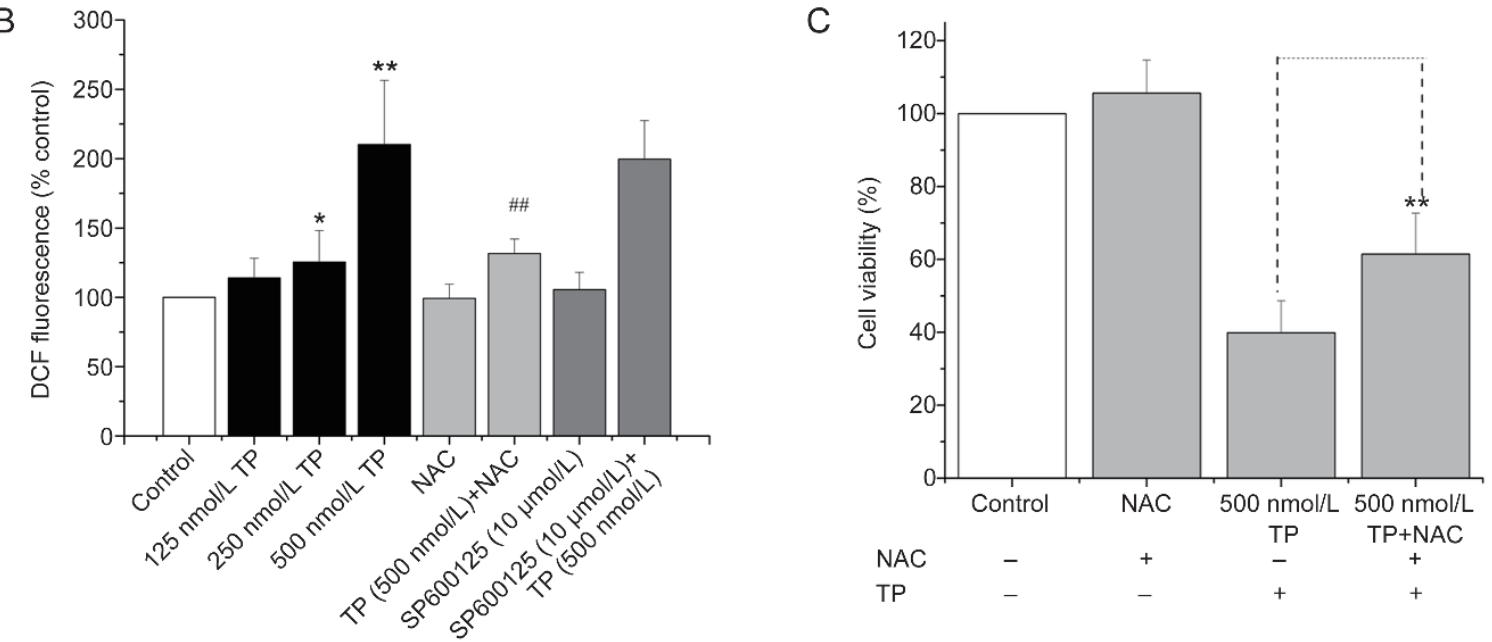

$\mathrm{E}$

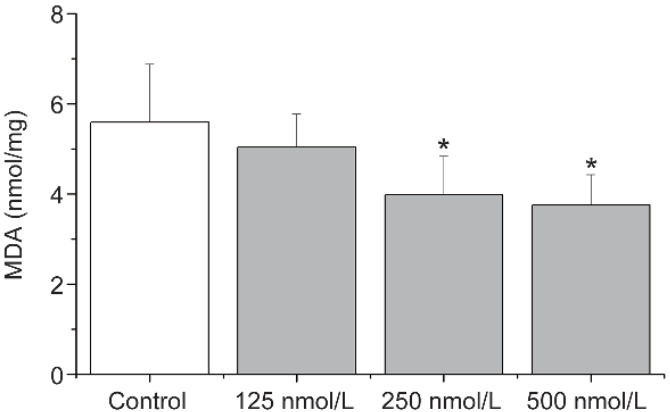

Figure 2. Role of ROS generation in TP-induced apoptosis in TM4 cells. (A) Intracellular ROS levels were examined using DCF fluorescence by fluorescence microscope. Cells were pretreated with different concentrations of TP $(125,250$, and $500 \mathrm{nmol} / \mathrm{L}), 500 \mathrm{nmol} / \mathrm{L} \mathrm{TP}+1 \mathrm{mmol} /$ L-acetylcysteine (NAC) and $\mathrm{H}_{2} \mathrm{O}_{2}(300 \mu \mathrm{mol} / \mathrm{L})$ for $3 \mathrm{~h}$, and incubated with DCFH-DA as the probe oxidized to DCF intracellular and emitting fluorescence. (B) The DCF fluorescence intensity was measured by a fluorescence spectrophotometer. (C) Effect of NAC on TP-induced cell death. Cell viability was estimated by MTT assay (data represent the mean \pm SD of six experiments in each group). (D) The MDA levels in culture medium of TP (125, 250, and $500 \mathrm{nmol} / \mathrm{L})$ treatment for $12 \mathrm{~h}$; (E) The intracellular SDH activations in TP $(125,250,500 \mathrm{nmol} / \mathrm{L})$ treatment for $12 \mathrm{~h}$. Data represent the mean $\pm \mathrm{SD}$ of three independent experiments. ${ }^{*} P<0.05,{ }^{* *} P<0.01$ vs untreated control cells. ${ }^{\# \#} P<0.01$ vs TP $(500 \mathrm{nmol} / \mathrm{L})$ cells. 

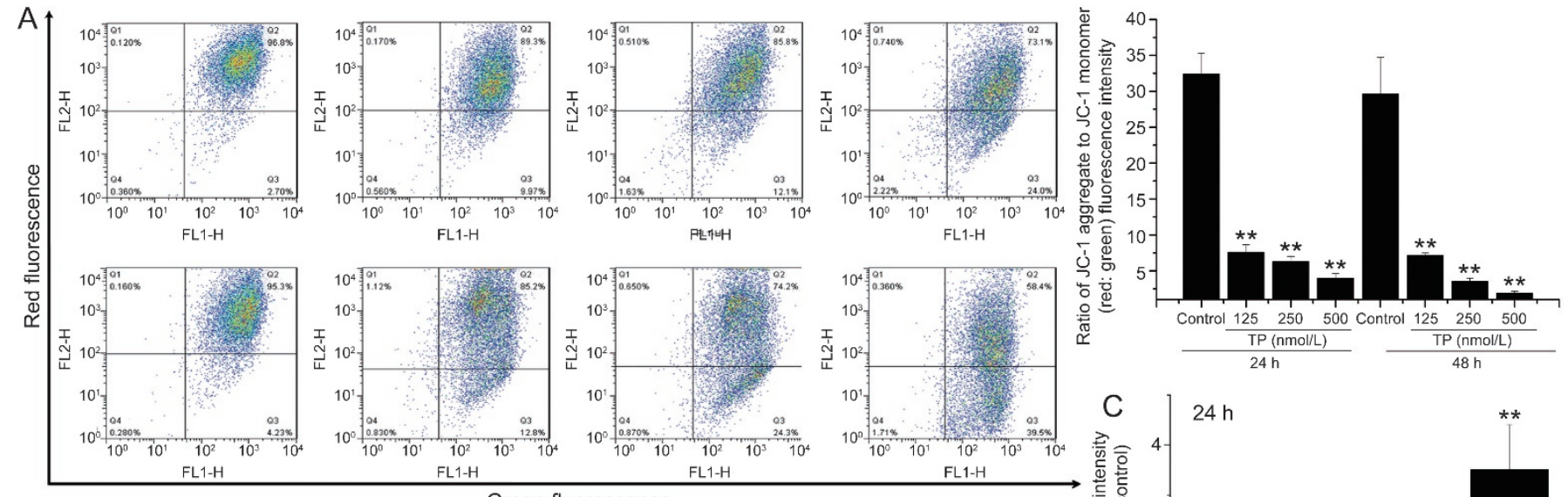

Green fluorescence

B

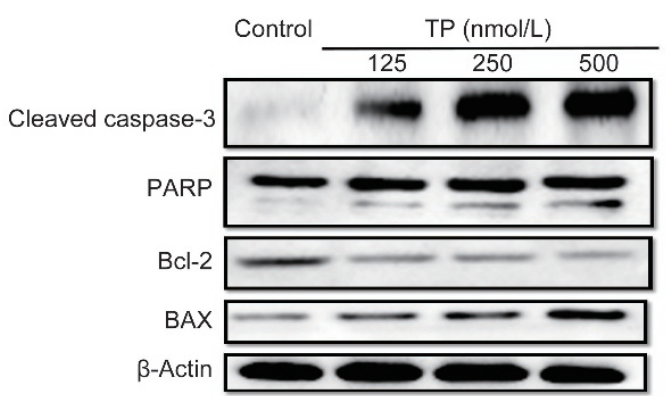

Cytochrome $c$

$$
\begin{gathered}
\text { Mitochondria } \\
\text { Cytoplasm }
\end{gathered}
$$

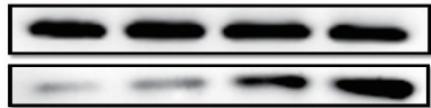

$24 \mathrm{~h}$
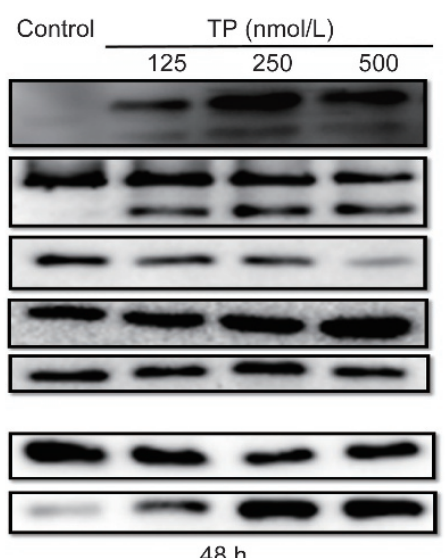
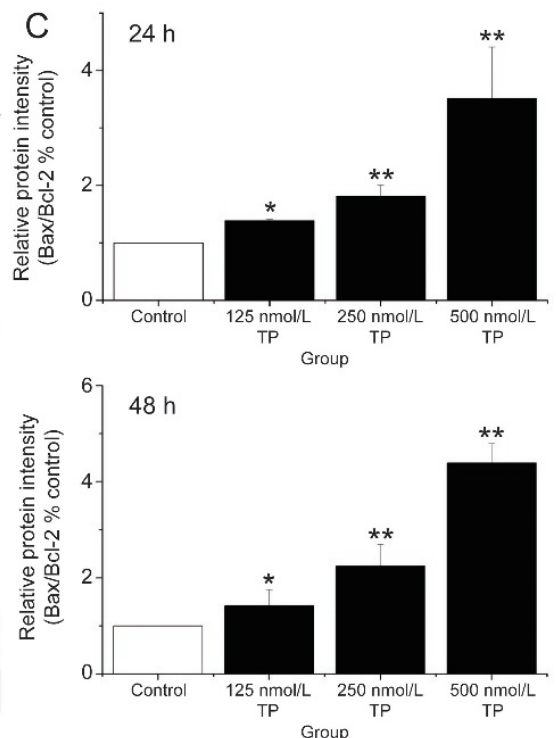

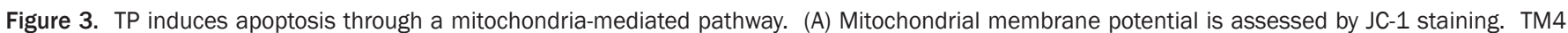

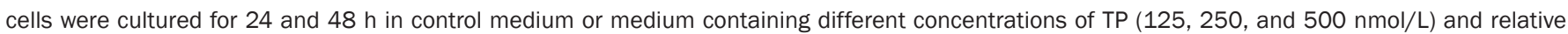

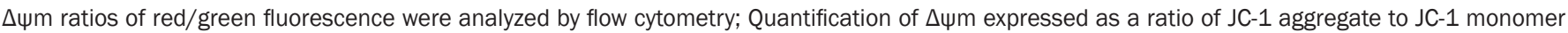

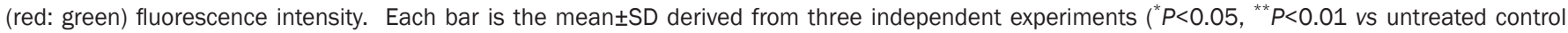

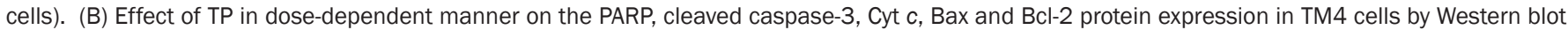

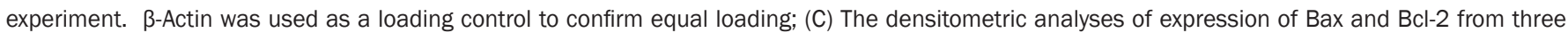
independent experiments. ${ }^{*} P<0.05,{ }^{* *} P<0.01$ vs untreated control cells.

\section{Effects of TP on the activation of MAPK signaling in cultured TM4 cells}

To assess the involvement of the MAPK signaling pathway in TP-induced apoptosis of SCs, the expression levels of both total and phosphorylated proteins were evaluated by Western blot analysis. TM4 cells were exposed to various TP levels $(125,250$, and $500 \mathrm{nmol} / \mathrm{L})$ for 24 and $48 \mathrm{~h}$. As shown in Figure $4 \mathrm{~A}$ and $4 \mathrm{~B}$, we observed activation of phospho-JNK in a dose-dependent manner, but observed no change in the total JNK concentration after TP treatment. Neither activation nor suppression was observed in the ERK1/2 and p38 signaling pathway in TM4 cells after TP treatment for 24 and 48 h. Furthermore, combined treatment with TP and the specific JNK inhibitor SP600125 directly inhibited TP-induced apoptosis of TM4 cells (Figure 4C). This phenomenon was not observed when the P38 inhibitor SB203580 and ERK inhibitor PD98059 were used. These results indicated that TP induces apoptosis in TM4 SCs by activating the JNK-MAPK pathway.
TP induces apoptosis in TM4 cells through ROS/JNK/mitochondriadependent apoptotic signals

We found that ROS and JNK activation play important roles in regulating TP-induced apoptosis in TM4 cells. This result was further confirmed by Annexin V-FITC/PI staining followed by flow cytometry (Figure $5 \mathrm{~A}$ ). The in vitro study showed that combined treatment with TP and SP600125 or NAC significantly decreased the ratio of early apoptotic cells induced by TP $(500 \mathrm{nmol} / \mathrm{L})$. In addition, both the loss of $\Delta \psi \mathrm{m}$ and the increase in apoptosis were significantly prevented by NAC and SP600125 (Figure 5B). As shown in Figure 5C, treatment with JNK inhibitor or NAC not only suppressed TP-mediated Bcl-2 down-regulation but also blocked the release of Cyt $c$ into the cytosol from mitochondria. Moreover, cleavage of caspase-3 and PARP determined by Western blot analysis provided further evidence that inhibitors of JNK and NAC suppress the activation of the effector caspase (Figure 5C).

Finally, we evaluated whether ROS, as an upstream signal, 
A

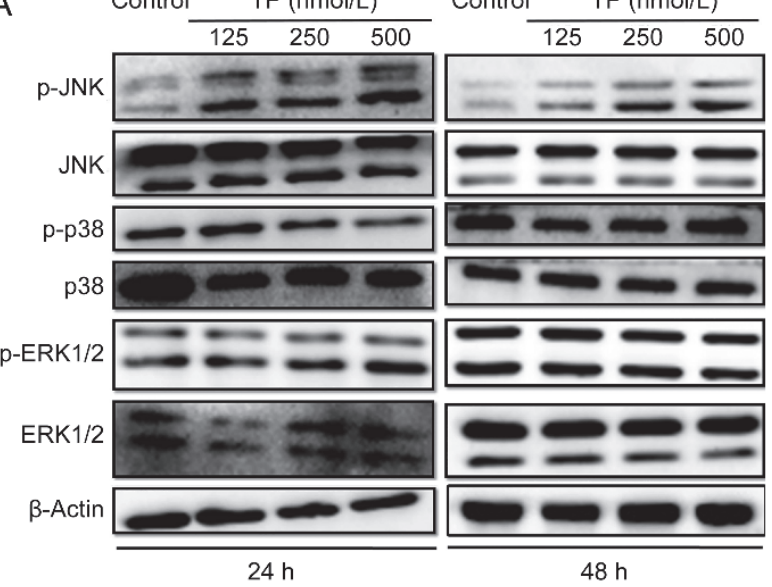

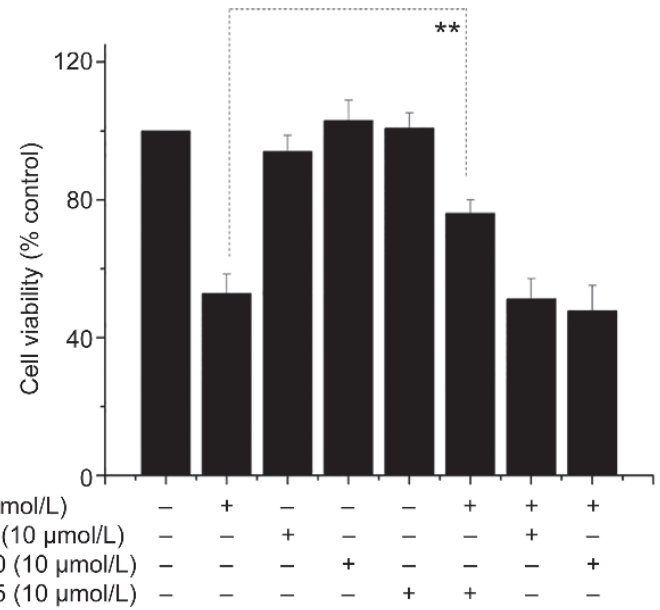

C PD98059 $(10 \mu \mathrm{mol} / L)$ SB203580 (10 $\mu \mathrm{mol} / \mathrm{L})$ SP600125 $(10 \mu \mathrm{mol} / \mathrm{L})$
$\mathrm{B}$

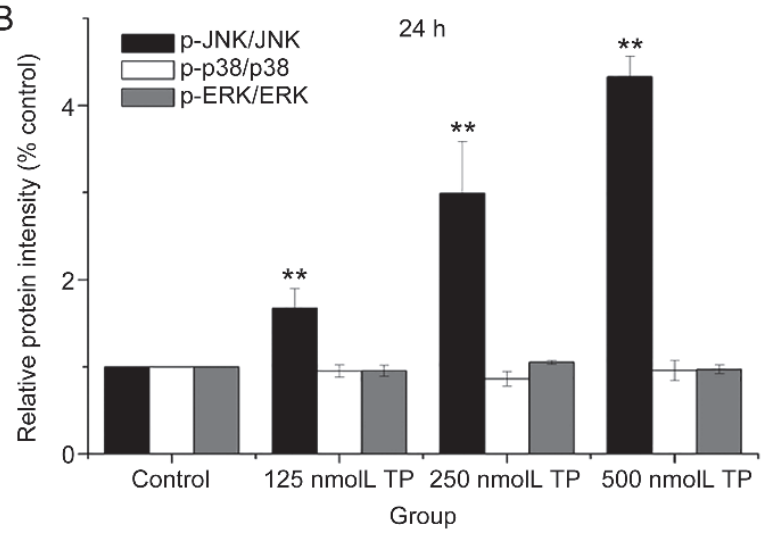

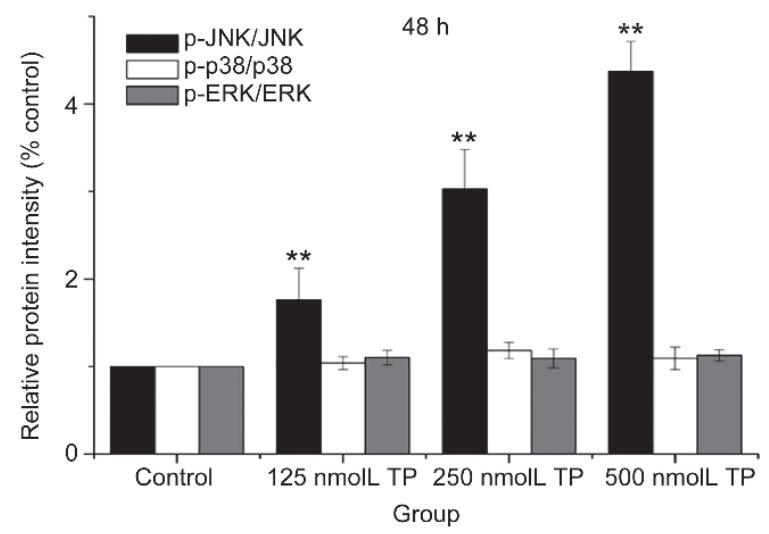

Figure 4. Activation of JNK phosphorylation during TP-induced apoptosis in cultured TM4 cells. (A) Cells were cultured in 6-well plates until confluent, and the medium was replaced with $1 \%$ serum medium in the presence or absence of TP $(125,250$ and $500 \mathrm{nmol} / \mathrm{L})$ for 24 and $48 \mathrm{~h}$. The cells were lysed and MAPKs proteins were analyzed Western blot. (B) Densitometry scanning analysis of ratio of p-JNK/total JNK, p-p38/total p38 and p-ERK1/2/ total ERK1/2. Data represent the mean \pm SD of three independent experiments. ${ }^{*} P<0.05,{ }^{* *} P<0.01$ vs untreated control cells. (C) Effects of JNK inhibitor SP600125, P38 inhibitor SB203580 and ERK inhibitor PD98059 on TP-induced apoptosis in cultured TM4 cells for 24 h. Cell viability was determined by MTT assay and data represent the mean \pm SD of six experiments in each group. ${ }^{*} P<0.05$ vs single TP treated cells.

can induce JNK activation and further trigger the mitochondrial apoptosis pathway. After Western blot analysis, we found that the ROS scavenging agent (NAC) inhibited JNK activation in TP $(500 \mathrm{nmol} / \mathrm{L})$-treated TM4 cells. Additionally, NAC, similarly to the specific JNK inhibitor SP600125, suppressed cell apoptosis by inhibiting JNK activation (Figure 5C). The data showed that ROS might be one of the upstream effectors of the JNK-mediated signaling pathway during TPinduced apoptosis of TM4 cells. TP-induced the generation of ROS and triggered apoptosis through JNK-dependent activation of the mitochondrial pathway.

TP prevented HO-1, NQO1 and CAT expression by inhibiting the activation of Nrf2

Nrf2 is an essential transcription factor that regulates the expression of genes involved in the cellular antioxidant defense system. To investigate whether TP could inhibit the transcriptional activation of certain antioxidant enzymes through the inactivation of Nrf2, RT-PCR of the relative RNA expression was performed. As shown in Figure 6A, TP caused a concentration-dependent and statistically significant decrease in the expression of HO-1, NQO1 and CAT mRNA expression. In addition, we did not observe clear nuclear translocation of Nrf2 by immunofluorescence analysis (Figure 6C) after stimulating the cells with TP. The translocation of Nrf2 from the cytoplasm to the nucleus was also detected by Western blotting. When TM4 cells were treated with TP $(125,250$, and 500 $\mathrm{nmol} / \mathrm{L}$ ) for $3 \mathrm{~h}$, the expression of nuclear Nrf2 was decreased, and there were no obvious changes in the expression of cytosolic Nrf2 (Figure 6D). TP prevented HO-1, NQO1 and CAT expression by inhibiting the activation of Nrf2. To confirm the important role of Nrf2 in regulating the TP-induced cytotoxicity of TM4, Nrf2 siRNA was used to knockdown the expression of Nrf2 in TM4 cells (Figure 6D). Compared with control siRNA, Nrf2 siRNA significantly decreased cell viability after treatment of TP (Figure 6F). Further evidence indicated that TP-induced excessive ROS production, potentially because of the lack of antioxidant gene stimulation in cells with Nrf2 knocked down by transient siRNA transfection (Figure 6G). Nrf2 siRNA markedly enhanced the rate of cell apoptosis after 
A

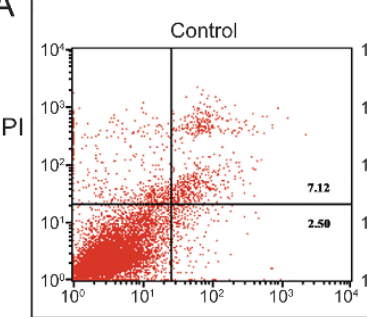

$500 \mathrm{nmol} / \mathrm{L} \mathrm{TP}$

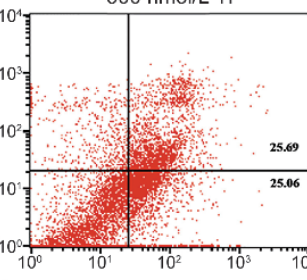

$\mathrm{NAC}+500 \mathrm{nmol} / \mathrm{L}$ TP

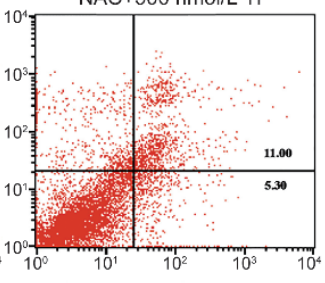

SP600125+500 nmol/L TP

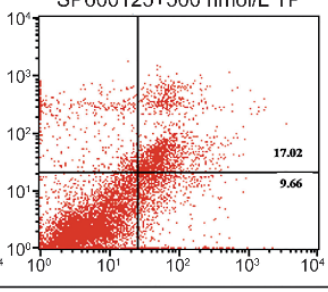

Annexin V-FITC

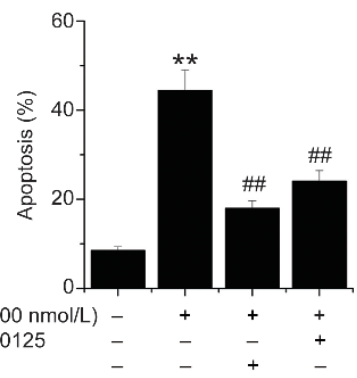

B

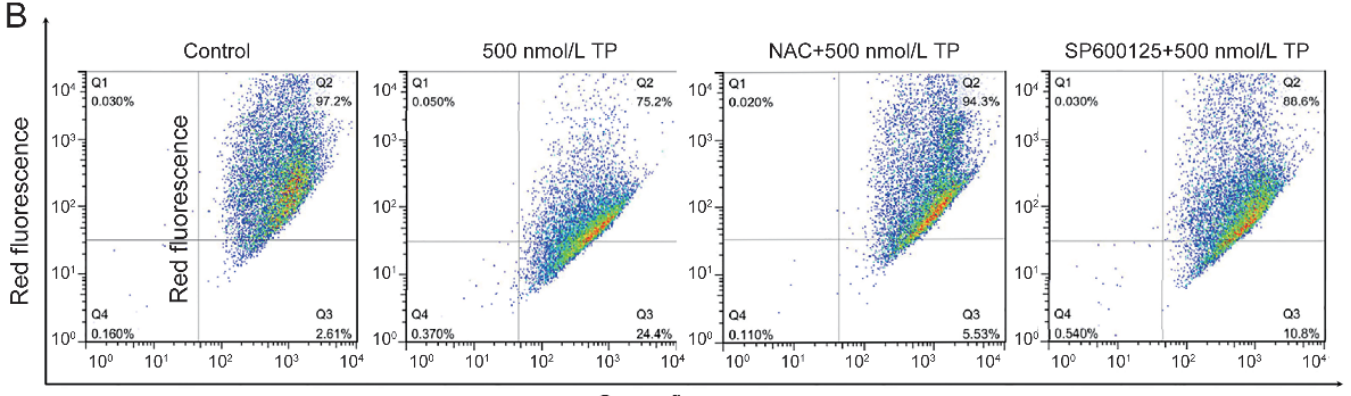

Green fluorescence

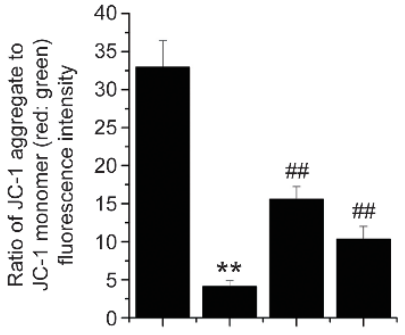

$\begin{array}{lllll}\mathrm{TP}(500 \mathrm{nmol} / \mathrm{L}) & - & + & + & + \\ \mathrm{SP} 600125 & - & - & - & + \\ \mathrm{NAC} & - & - & + & -\end{array}$

C

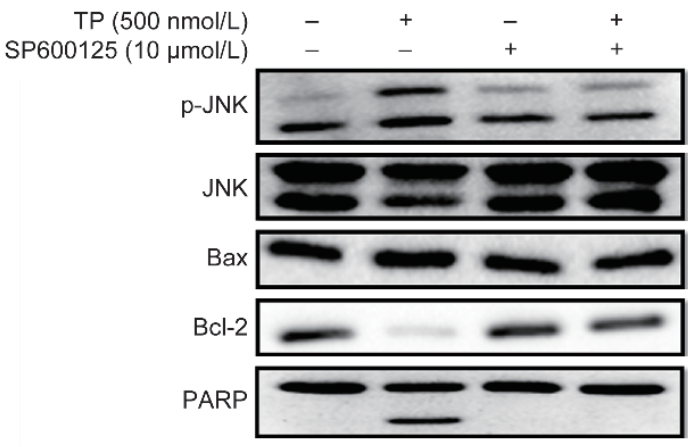

Cleaved caspase-3

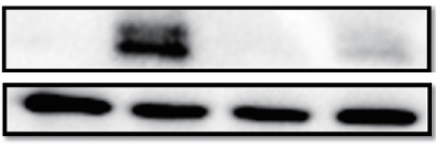

$24 \mathrm{~h}$
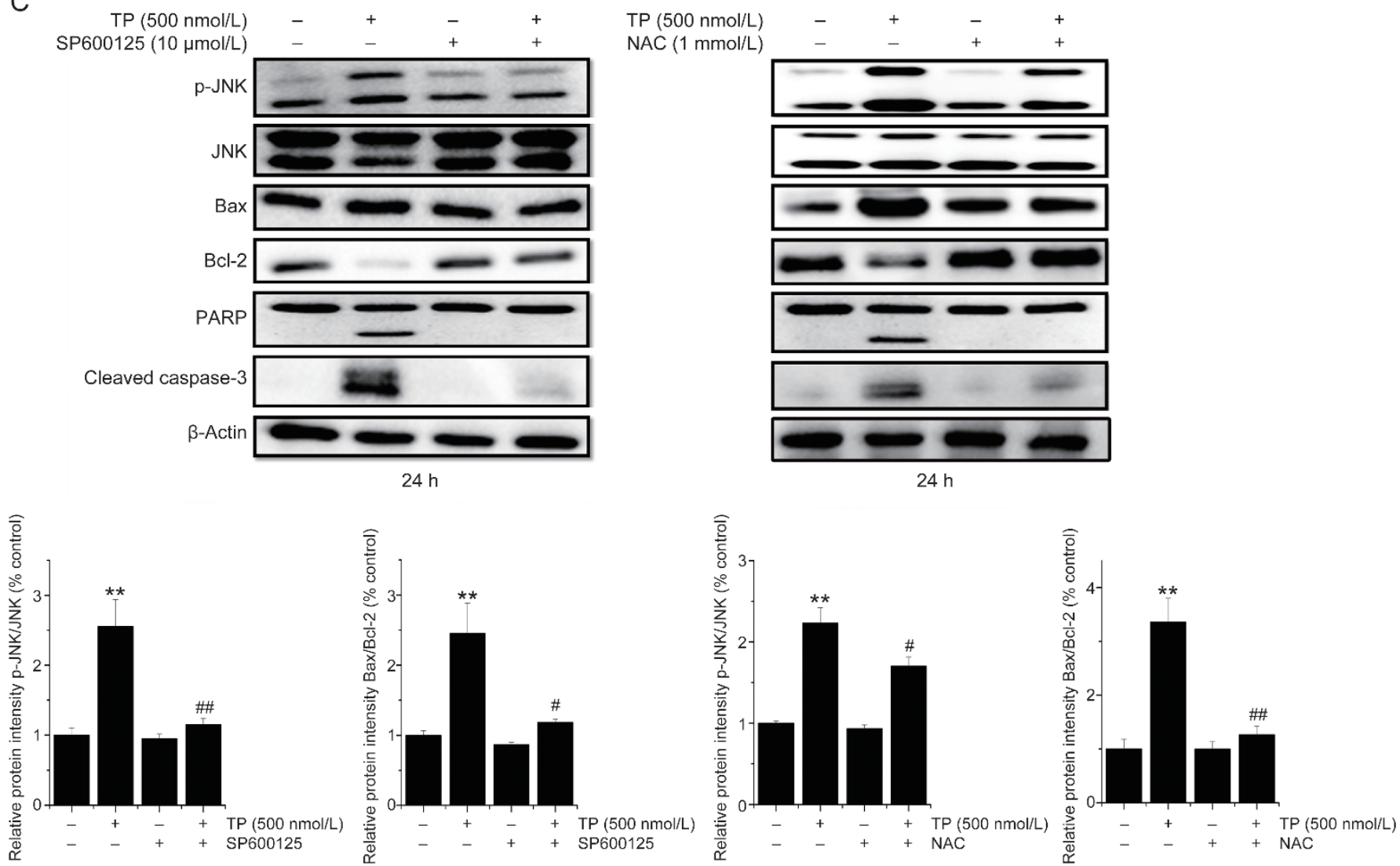

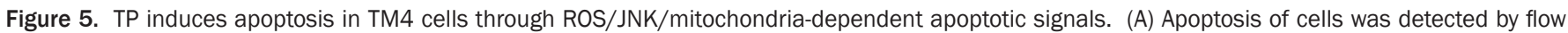

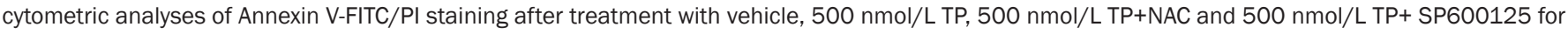

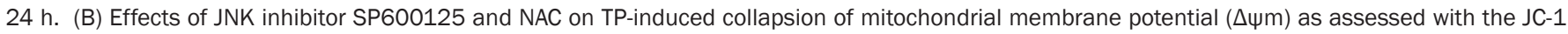

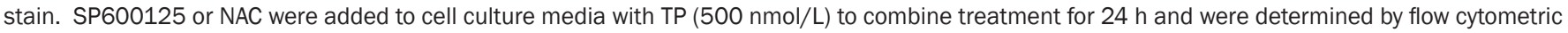

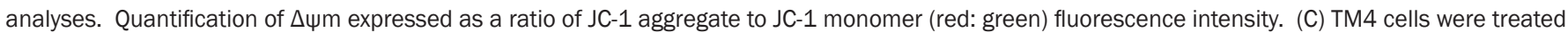

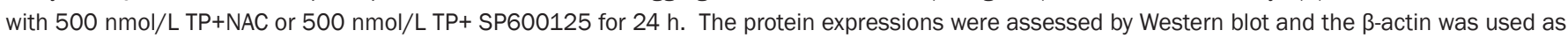

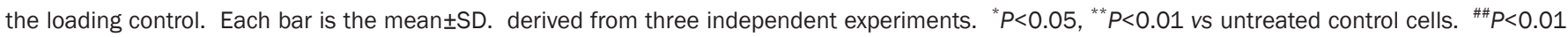
as compared with TP group. 

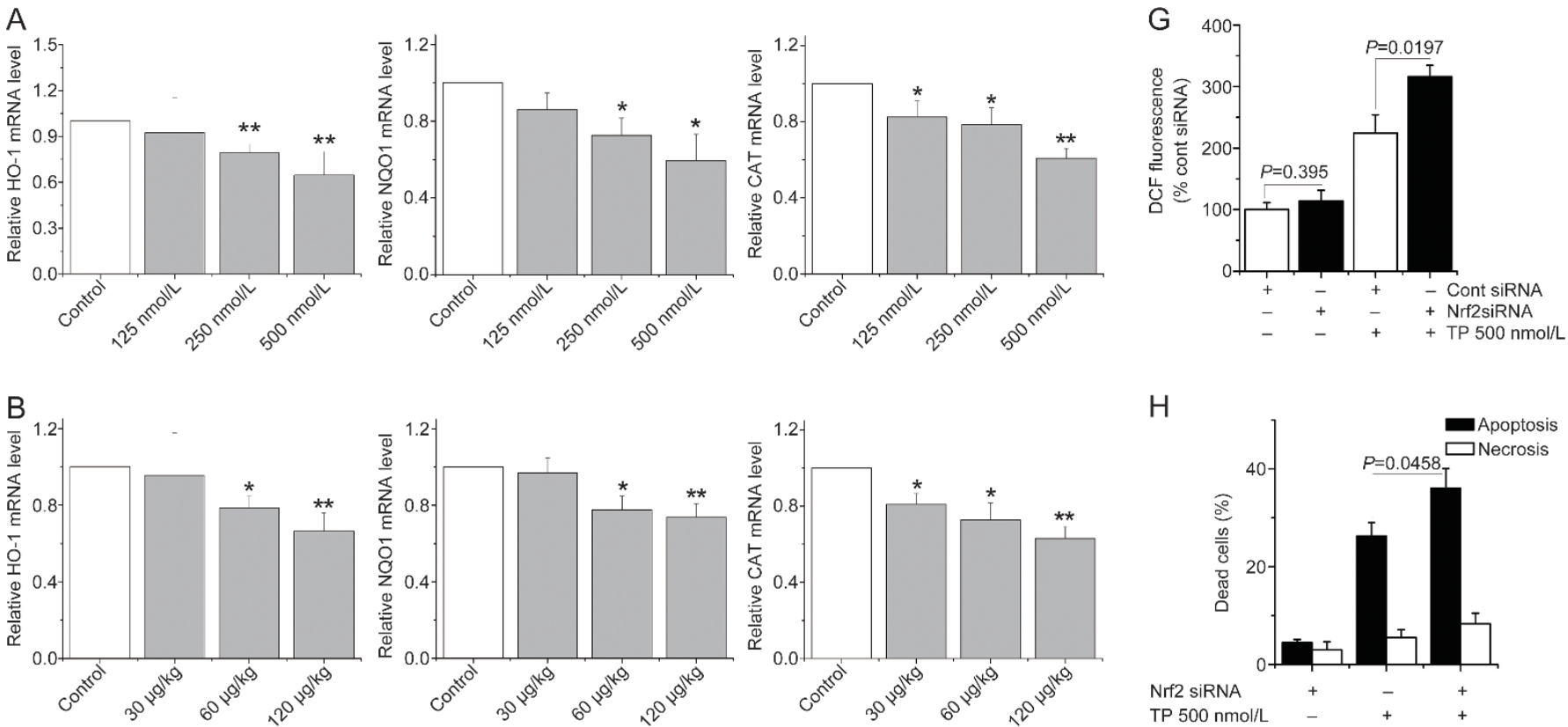

$\mathrm{H}$

C
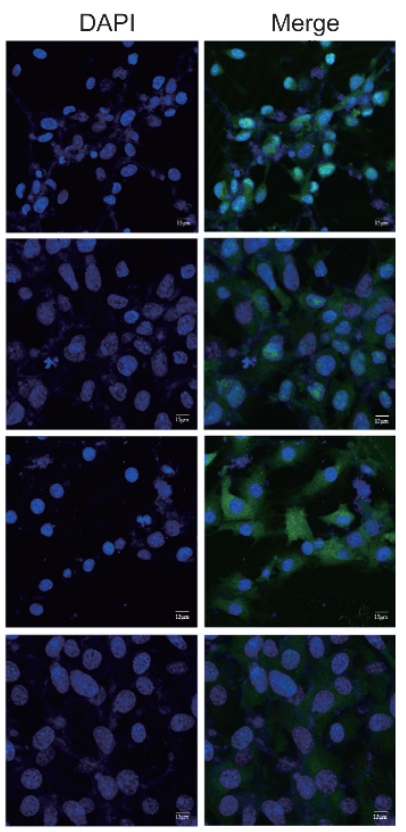
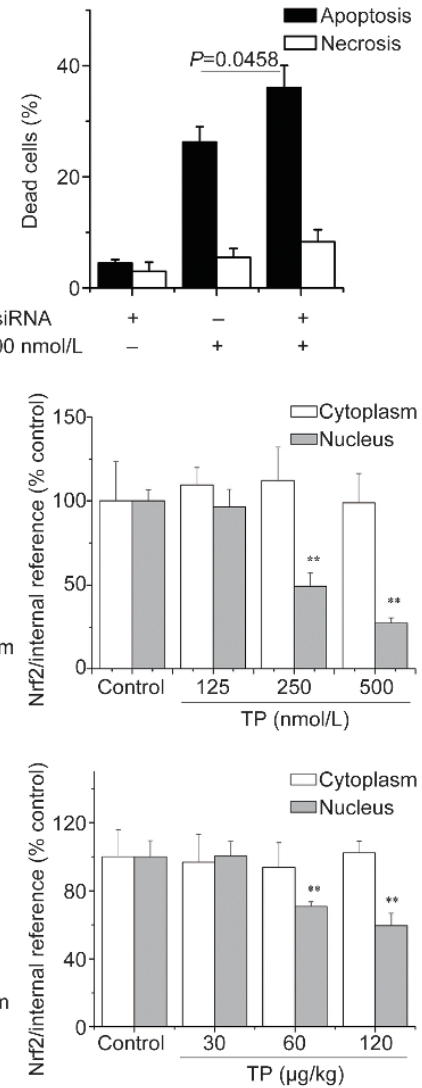

Nrr2 SiRNA

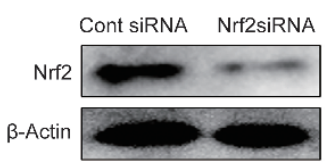

D

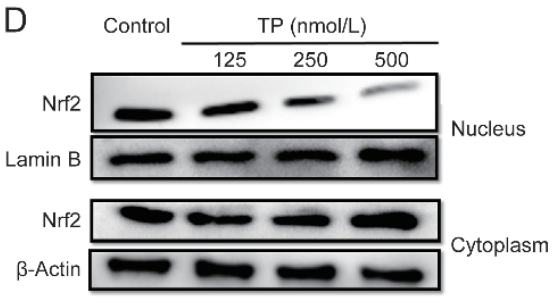

$\mathrm{E}$
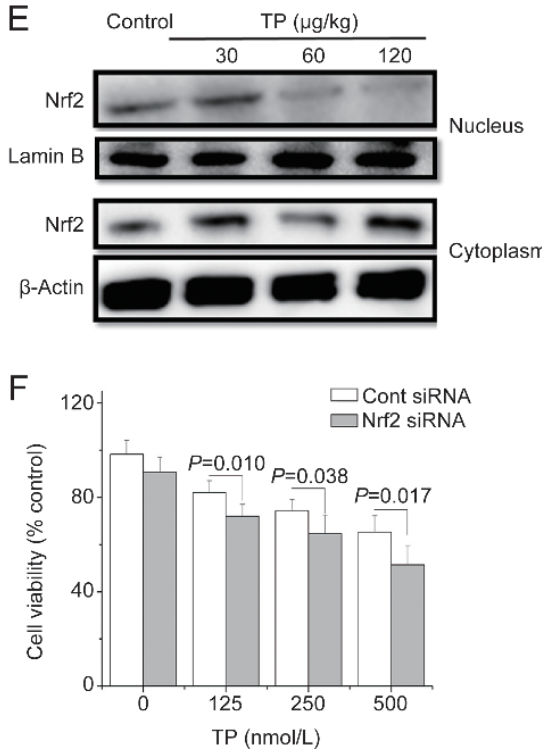

Figure 6. TP inhibit the transcriptional activation of Nrf2. The mRNA expressions of HO-1, NQO1 and CAT were detected by Real time PCR in TM4 cells (A) and testicular tissues (B). Data represent the mean \pm SD of three independent experiments. ${ }^{*} P<0.05,{ }^{* *} P<0.01$ vs untreated control group. (C) Effects of TP-inhibit Nrf2 nuclear translocation analyzed by immunofluorescence staining. (D) TM4 cells were treated with TP (125, 250 and $500 \mathrm{nmol} / \mathrm{L})$ for $6 \mathrm{~h}$. The translocation of Nrf2 from cytoplasm into nucleus was detected by Western blot as well as $\beta$-actin and LaminB were used as loading control for cytoplasm or nucleus, respectively. (E) Representative blots of nuclear and cytosolic Nrf2 in testicular tissues; The $\beta$-actin and Lamin B were used as the loading control for cytoplasm or nucleus, respectively. (F) Influence of Nrf2 knockdown on the effect of TP induced cell death measured by MTT. (G) Influence of Nrf2 knockdown on the effect of TP induced accumulation of ROS was measured by a fluorescence Spectrophotometer. (H) Influence of Nrf2 knockdown on the effect of TP induced apoptosis in TM4 cells by Annexin V-FITC/PI binding and measured by flow cytometry analysis. The histogram illustrates apoptosis proportion from three separates experiments. 
treatment with TP (Figure 6H). These data suggested that Nrf2 is directly involved in TP-induced cytotoxicity of TM4.

\section{TP-induced testicular injury in male mice}

To evaluate whether the toxic effects of TP on testis damage and male infertility could be explained by the induction of apoptosis, detection of biochemical indicator and analysis of histopathology and apoptosis of the testicular tissue of male mice were performed after TP treatment for 14 days. Above all, significant differences were observed for the testes index (testes weight/body weight) between the mice that received oral administration of TP $(30,60$ and $120 \mu \mathrm{g} / \mathrm{kg})$ daily for 14 days and the mice that were treated with normal saline, thus suggesting that TP was severely toxic to testicular tissues of mice in vivo (Figure 7A). SDH was distributed in the inner mitochondrial membrane and played an important role in supplying energy for sperm maturation. The activities of SDH after treatment with 30,60 and $120 \mu \mathrm{g} / \mathrm{kg}$ TP were significantly decreased to approximately $20 \%, 34 \%(P<0.05)$ and $44 \%$ $(P<0.05)$ of the levels in the control group on day 14 , respectively (Figure 7C). Decreased SDH levels in testicular tissue not only led to abnormal sperm (Supplemental material Figure S3) but also reflected the collapse of the energy supply from $\mathrm{SC}$ and abnormal mitochondrial function. MDA is a lipid peroxidation by-product that is an indicator of cellular oxidation status. MDA levels in mice treated with $60(P<0.05)$ or $120(P<0.01) \mu \mathrm{g} \cdot \mathrm{kg}^{-1} \cdot \mathrm{d}^{-1} \mathrm{TP}$ were markedly increased as compared with levels in the control group (Figure 7B). Moreover, a marked decrease in SOD activity in testicular tissue was observed in the high-dose group $(P<0.01)$ compared with the control group (Figure 7D). As shown in Figure 7G, the testicular tissues of the mice in the TP $(30,60$ and $120 \mu \mathrm{g} / \mathrm{kg})$ groups displayed abnormal histopathological changes compared with those in the control group. TP $(120 \mu \mathrm{g} / \mathrm{kg})$ led to a substantial decrease in the internal diameter of the seminiferous tubules, damage to the basement membrane and scant cytoplasm, as well as sperm cell shedding from SCs. In addition, the effect of TP on the mRNA level of FSHR in testis was also studied. The mRNA levels of FSHR in testis were down-regulated in a dose-dependent manner in TP-treated animals, compared with control animals (Figure 7F).

Caspase- 3 activity in testicular tissue was also analyzed to confirm the contribution of caspase activation to the induction of apoptosis in vivo. As shown in Figure 7E, caspase-3 activity in testicular tissues in the three TP groups increased significantly in a dose-dependent manner, as compared with the activity in the control group. We further report the first investigation of DNA fragmentation in the testicular tissues of male mice by using TUNEL staining after TP or vehicle treatment for $14 \mathrm{~d}$. The TUNEL assay explicitly showed that positive cells (apoptotic cells) were induced by TP in testicular tissue and that almost no positive cells were detected in the control group (Figure 7H). Most TUNEL-positive cells had some morphological characteristics of SCs. The effect of TP on $\mathrm{p}$-JNK expression was detected in mouse testicular tissue by immunocytochemistry. As shown in Figure 7I, the immu- nohistochemical staining analysis exhibited a concentrationdependent elevation in p-JNK expression.

We further explored the transcriptional function of Nrf2 by detecting the expression of its downstream target antioxidant genes at the mRNA levels. The data (Figure 6B) indicated that HO-1 $(P<0.05, P<0.01)$, NQO1 $(P<0.05, P<0.01)$ and CAT mRNA $(P<0.05, P<0.01)$ were markedly decreased after TP $(60$ and $120 \mu \mathrm{g} / \mathrm{kg}$ ) treatment. Our results showed that the testis tissue from TP-treated mice exhibited decreased nuclear translocation of Nrf2 (Figure 6E). The results of different assays using different methodology strongly suggested that TP caused severe toxicity to testicular tissue, especially by inducing SC apoptosis, thus finally resulting in testis damage and subsequent male infertility.

\section{Discussion}

Previously, we have reported that TP causes testicular damage and depletion of the energy supply from SCs, thus leading to decreases in sperm count and motility and abnormal changes in sperm morphology in male mice. Although it has been shown that abnormal sperm are derived from testicular tissue damage, the mechanisms underlying TP-induced apoptosis of SCs remain obscure. The role of TP in SC apoptosis and its underlying mechanism of action have not been previously reported. In previous studies, TM4 cells have been used as a mouse SC model to investigate the potential reproductive toxicity of, compounds such as bisphenol A, ethanol and nonylphenol. In the present study, we performed a series of in vitro and in vivo experiments to comprehensively evaluate the molecular mechanisms of TP-induced apoptosis of TM4 cells. The results of our in vitro mechanistic studies revealed that TP promotes ROS production, thus further activating the JNK pathway and inhibiting the Nrf2 pathway. These pathways subsequently lead to inactivation of the antioxidant system, thus further triggering the mitochondrial-mediated apoptosis signaling pathway. Our further in vivo experiments showed that TP decreased testicular weight, increased MDA levels, destroyed the microstructure of the testis, promoted apoptosis of the testicular tissue, disrupted the mitochondrial enzyme activity and activated JNK phosphorylation in vivo.

It has been well documented that TP-induced organ toxicity is closely associated with increasing ROS levels in organs such as the liver, kidney, and heart ${ }^{[2,11,34]}$. However, there is little information available regarding the mechanism(s) underlying the toxic effects of TP on SCs. In our experiment, a dramatic ROS burst was observed during TP-induced apoptosis of TM4 cells, a result indicating that ROS accumulation is involved in TP-induced apoptosis in TM4 cells; however, the free radical scavenger NAC prevented this phenomenon. However, many studies have reported that TP-mediated regulation of ROS might be cell-type specific. TP inhibits PC12 cell apoptosis and provides potential therapeutic effects in Alzheimer's disease (AD) by down-regulating the generation of ROS, hydrogen peroxide $\left(\mathrm{H}_{2} \mathrm{O}_{2}\right)$ and MDA levels ${ }^{[22]}$. In a murine peritoneal macrophage cell line, TP inhibits the production of superoxide anion by attenuating oxidative stress and regulating inflam- 
A
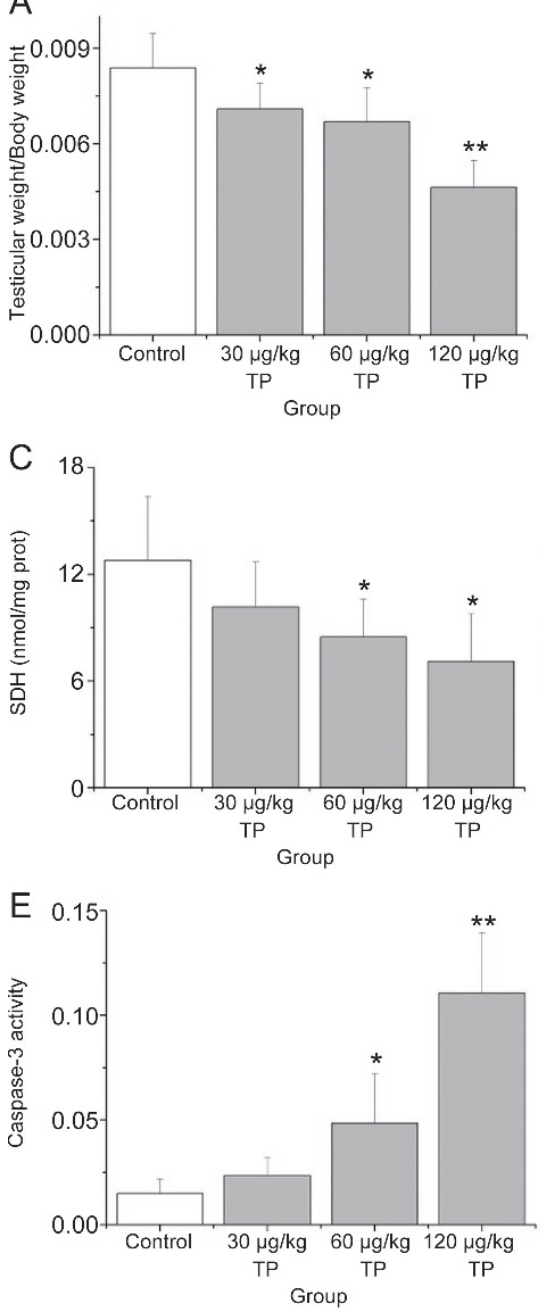

B

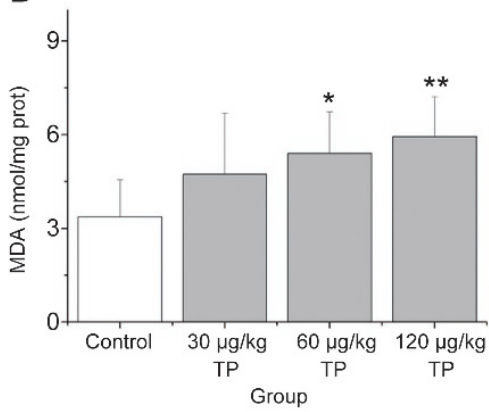

D
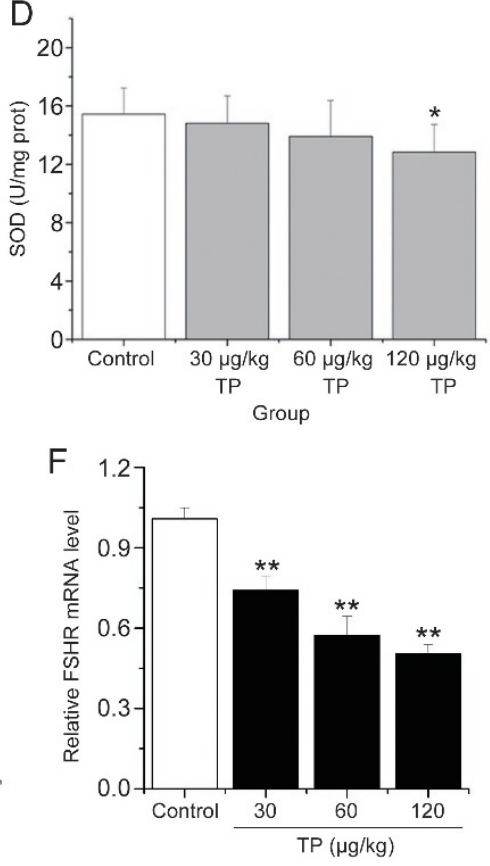

G

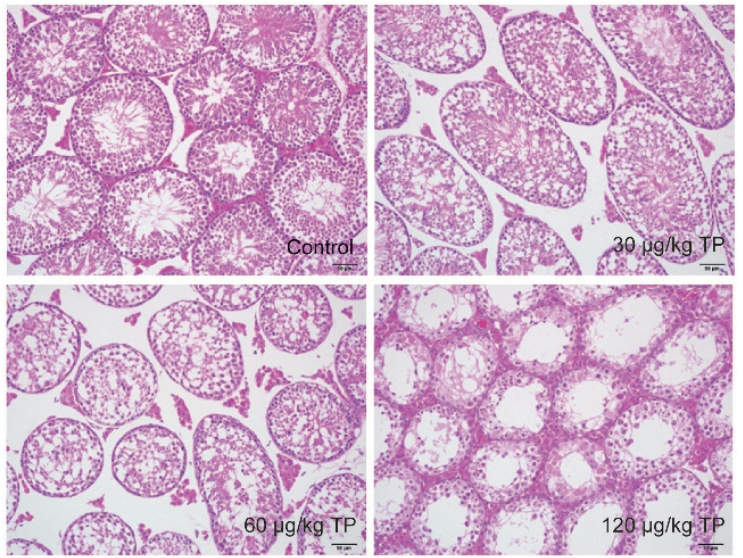

$\mathrm{H}$

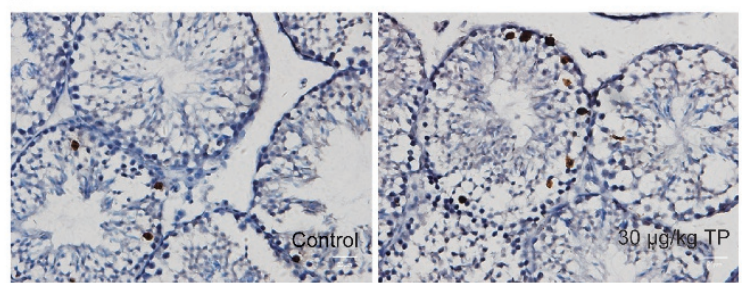

I

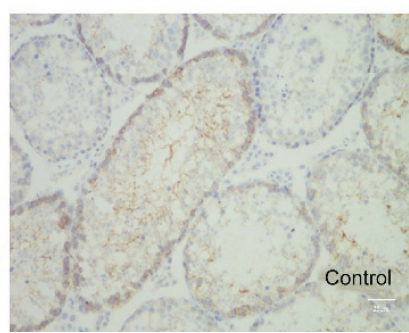

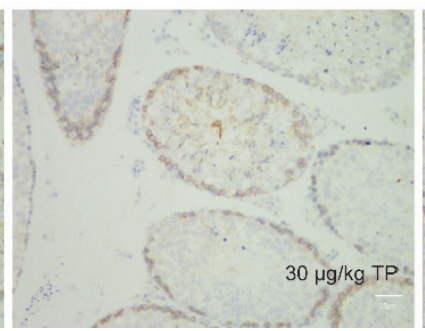

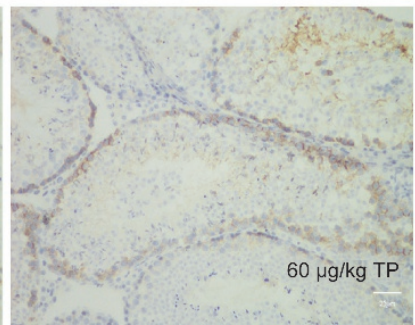

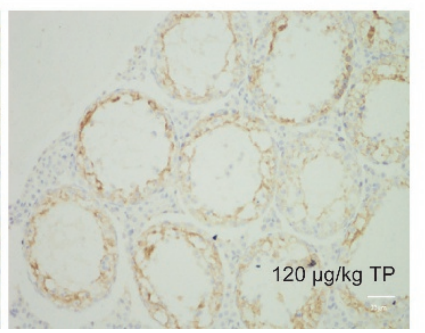

Figure 7. TP induced testicular injury in mice. (A) The testis indexes of mice after the treatment of TP for 14 days; (B) The MDA levels in testicular tissue; (C) The SDH activations in testicular tissue; (D) The SOD activations in testicular tissue; (E) Activations of caspase-3 in testicular tissue were determined by classical colorimetric method by commercial kits; (F) The mRNA expression of FSHR was detected by real-time PCR in testicular tissues. Data represent the mean \pm SD of eight independent experiments. ${ }^{*} P<0.05,{ }^{* *} P<0.01$ vs untreated control. (G) Histological analysis [stained with $\mathrm{H} \& \mathrm{E} 200 \times$ ] of testicular tissue in mice induced by TP for 14 days. (H) In situ detection of apoptotic cells in testis sections after the treatment of TP for 14 days by optical microscope using the TUNEL assay (normal cells: bluish violet; apoptotic cell: brown). (I) Effect of TP on p-JNK expressions in male mice testis sections were detected by immunocytochemistry (positive cell: claybank).

mation through NF-kB-mediated inhibition of inflammationrelated cytokines ${ }^{[35]}$. In contrast, TP promotes ROS production and decreases $\Delta \Psi_{\mathrm{m}}$ levels in human adrenal cancer NCI-H295 cells ${ }^{[36]}$. This contradictory mechanism of TP has also been reported in other studies ${ }^{[1,2]}$. Therefore, our in vivo study was carried out to confirm whether ROS accumulation is the main mechanism underlying the TP-induced apoptosis of SCs and testicular injury. In our experiments, MDA and SOD were assessed as key indicators of oxidative stress in testicular tissue. MDA is one of the most widely used indicators of free radical formation and is formed by ROS degradation of polyunsaturated lipids. Moreover, superoxide dismutase (SOD) is a critical antioxidant enzyme that removes the ubiquitous superoxide metabolic products in biological systems. Sig- 


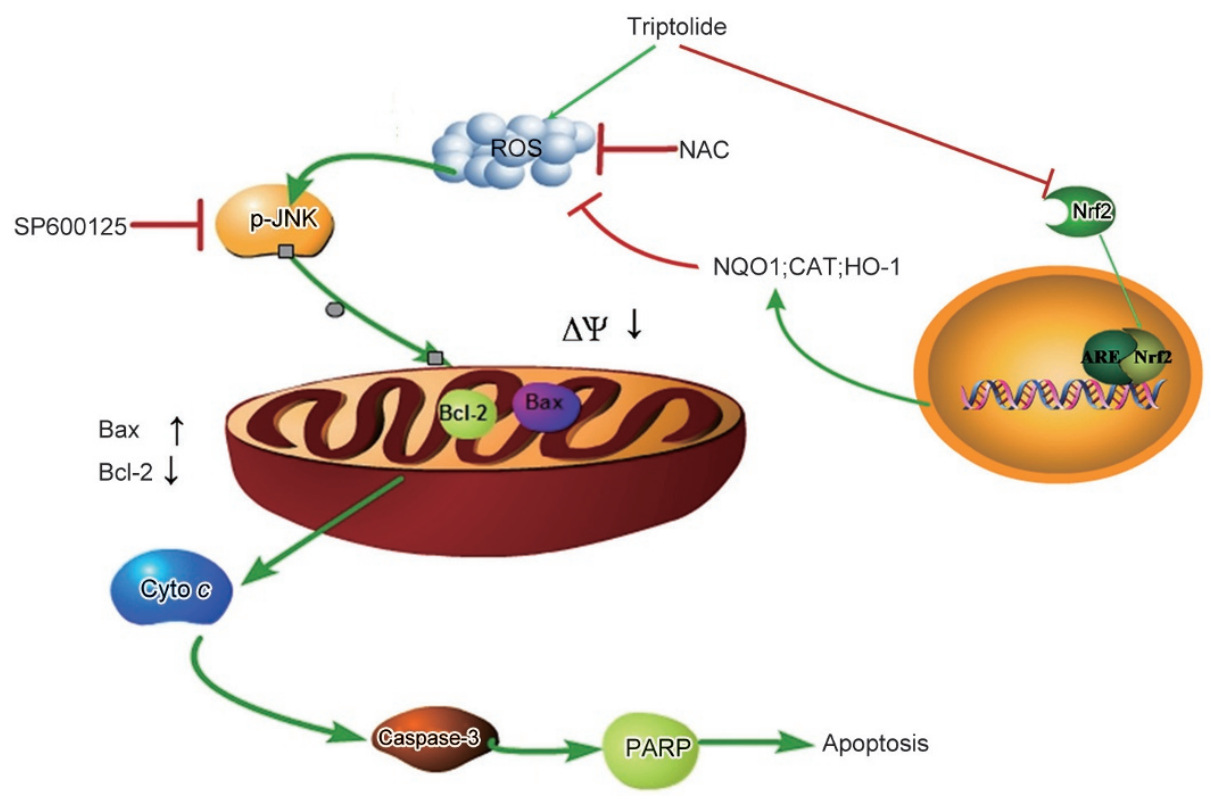

Figure 8. Overview of pathways for TP induced apoptosis of SCs.

nificantly increased levels of MDA and decreased activities of SOD observed in testicular tissue after TP administration indicated that TP led to severe oxidative damage in vivo. The results suggested that TP triggers apoptosis of SCs or testicular injury at least in part by inducing ROS accumulation.

Nrf2 is a pivotal player in the cellular antioxidant defense system. Our results suggested that TM4 cells and testis tissues after TP treatment exhibited decreased nuclear translocation of Nrf2, thus potentially leading to weaker induction of antioxidant defenses including a decrease in the activity of GSH and the gene expression of NQO1, CAT and HO-1. Therefore, the TP-induced oxidative stress damage to TM4 cells may partly rely on its Nrf2 inactivation-mediated suppression of antioxidant activities. A similar mechanism of action has been identified in Leydig cells. Zhang and co-workers have suggested that TP inhibits the expression of HO-1 and downstream Nrf2 activation, thus leading to further apoptosis of Leydig cells (the steroidogenic cells located in the interstitium of the testis $)^{[37]}$. TP led to a loss of Nrf2-mediated cytoprotective responses under persistent oxidative stress conditions.

Mitochondria are mainly responsible for energy metabolism in cells, representing one important source of cellular ROS generation and regulation of apoptosis. In mitochondrialdependent apoptotic signaling, cell apoptosis is regulated by pro- and anti-apoptotic proteins. The anti-apoptotic protein Bcl-2 localized in the mitochondrial wall regulates apoptosis by controlling mitochondrial permeability and inhibiting Cyt $c$ release. The pro-apoptotic Bax protein enhances the release of Cyt $c$ by translocating to mitochondria from the cytosol after death signaling. Elevated ROS accumulation activates mitochondrial-dependent apoptotic signaling by increasing the permeability of the outer mitochondrial membrane and further induces apoptosis through the activation of caspase cascades.
Whether mitochondrial dysfunction plays an important role in apoptotic events in SCs remains unclear. Our results showed that TP-induced a decrease in mitochondrial membrane potential, attenuated expression of the anti-apoptotic protein Bcl-2, and increased the expression of the pro-apoptotic protein Bax, thereby eventually increasing the Bax/Bcl-2 ratio and promoting the protein expression of Cyt $c$ in the cytoplasm, and leading to effector caspase activation and subsequently apoptosis. Caspase- 3 activation is a central feature of apoptotic cells. Increases in protein expression of both cleaved caspase- 3 and PARP were observed during TP-induced apoptosis of SCs. More importantly, our data showed that combination treatment with TP and NAC dramatically reversed the loss of $\Delta \psi_{\mathrm{m}}$ and decreased the release of Cyt $c$, activation of caspase-3, and the percentage of apoptosis, thus indicating that intracellular ROS accumulation plays a key role in inducing mitochondrialdependent apoptotic signaling and results in TM4 cell apoptosis. SDH is also a marker that reflects mitochondrial function, and its suppression can be used as a proxy for testicular energy metabolism and spermatic function in vivo. In the present study, we observed depressed SDH activity, increased MDA levels and decreased SOD activity, which also reflected the oxidative damage to mitochondria. Our in vivo experiments also showed that apoptosis of testicular tissues resulted in additional severe tissue lesions, as assessed by the testis histopathology.

The MAPK signaling cascade was originally identified as a key pathway in the transduction of apoptotic signals. Previous studies have demonstrated that the toxicity and pharmacological activity of TP or GTW are closely related to MAPK signaling activation. Tan and co-workers have suggested that ERK1/2 activation and the generation of ROS are pivotal in regulating TP-induced apoptosis in MDA-MB-231 breast 
cancer cells ${ }^{[34]}$. TP induces apoptosis of dendritic cells by upregulating p38 phosphorylation ${ }^{[21]}$. In contrast, MAPKs are not involved in TP-induced cell apoptosis in prostate cancer cell lines even if TP can activate the MAPK signaling pathway $^{[34]}$. In the present experiments, TP-induced apoptosis of TM4 mouse SCs by activating the phospho-JNK in a dosedependent manner. Additionally, combination treatment with TP and the specific JNK inhibitor SP600125 significantly decreased TP-induced apoptosis and enhanced survival of TM4 cells, thus suggesting that JNK, but not p38 and ERK1/2, participates in TP-induced TM4 apoptosis.

JNK is known to be involved in the induction of many types of apoptosis. For example, JNK plays a key role in TNF-a-, Fas ligand-, X-ray- and UV ray-induced apoptosis. Furthermore, JNK is a primary regulatory factor associated with cell apoptosis when SCs are exposed to reproductive toxicants such as BPA, chromium chloride and 1, 3-DNB ${ }^{[31]}$. ROS serve as a strong signal for the activation of JNK and are an important regulatory factor in JNK-dependent apoptosis regulation $^{[38]}$. ROS are a potent activator of JNK through inhibition of oxidative endogenous JNK inhibitors ${ }^{[39]}$. Indeed, TPmediated JNK phosphorylation and ROS accumulation are all inhibited by NAC, thus indicating that the accumulation of ROS occurs upstream of the control signal, thereby resulting in sustained JNK activation and leading to cell death. The results of previous studies have revealed that induction of apoptosis by activated JNK is essential in modulating the functions of pro- and anti-apoptotic proteins located in mitochondria ${ }^{[40,41]}$. MAPK-signaling pathways have been shown to play vital roles in mitochondrial-mediated apoptosis. The relationship between mitochondrial apoptosis and the JNK pathway has also attracted the attention of many researchers. Bax is been shown to be a JNK substrate in several studies. JNK has also been reported to modulate the downstream activities of the pro-apoptotic Bcl-2 family, release of Cyt $c$ from mitochondria and extrinsic apoptotic pathways. Lei et al have indicated that defects in apoptosis are associated with a failure of JNK-deficient cells to release cytochrome $c$ (mitochondrial pro-apoptotic protein $)^{[42]}$. However, in another mechanism, caspasedependent mitochondrial dysfunction and activation of the MAPK pathways are independent pathways ${ }^{[43]}$. In the present research, this apoptotic activity was found to be partially blocked by the specific JNK inhibitor SP600125, thus providing further confirmation that the mitochondrial apoptotic pathway was regulated by the JNK signaling pathway. Therefore, activation of phospho-JNK may be a potential mechanism for the TP-induced mitochondrial apoptosis of SCs.

Our results indicated that combination treatment of TP with NAC or JNK inhibitor notably abolished TP-induced apoptosis, as evidenced by a decrease in PARP cleavage, an increase in cell viability and the recovery of $\triangle \Psi \mathrm{m}$ in TM4 cells. In addition, TP-mediated JNK activation was inhibited by NAC, thus indicating that JNK phosphorylation and the mitochondrial pathway are critical for TP-mediated apoptosis in TM4 cells, in which ROS acts as an important upstream mediator. In addition, the ability of TP to inhibit the Nrf2 pathway, which alters the appropriate functioning of the body's defense system, may be an important mechanism in the induction of TM4 cell apoptosis (Figure 8). We also observed that NAC and SP600125 significantly attenuated TP-induced apoptosis of TM4 cells, but those inhibitory effects were not very substantial. Therefore, it is likely that other mechanisms (inflammatory cytokines, Fas/ Fasl, energy metabolism, among others) that are distinct from oxidative stress may be involved in TP-induced apoptosis of TM4 cells. However, it is reasonable to speculate that the TPinduced apoptosis of TM4 cells and testis injuries observed in the present study occurred, at least in part, through ROS/ JNK-dependent activation of the mitochondrial pathway. Our results also suggested that co-administration of TP with antioxidants to prevent testicular injury may be a promising method to avoid the toxicity of TP in clinical therapy. Further studies are warranted to determine more precise mechanisms of TP-induced apoptosis of SCs and to screen for suitable antioxidants in vitro and in vivo.

Our present study comprehensively investigated the antiproliferative effect of TP on TM4. We report the first evidence that TP-mediated testicular toxicity is mediated by activation of the mitochondrial death pathway in Sertoli cells via ROS/ JNK activation and Nrf2 pathway suppression. These results provide important new insights into the toxic molecular pathways of TP and the mechanism of testis diseases. Our data also indicated that combining TP with a ROS scavenging agent may be a promising strategy to reverse TP-mediated reproductive toxicity.

\section{Abbreviations}

TP, Triptolide; NAC, N-acetyl cysteine; ROS, reactive oxygen species; SDH, succinodehydrogenase; MAPKs, mitogen activated protein kinases; GTW, Tripterygium wilfordii multiglycoside; SCs, Sertoli cells; JNK, c-Jun N-Terminal kinase; MDA, malondialdehyde; Cyt c, Cytochrome c; DCFDA, 6-Carboxy-2', 7 '-dichlorofluorescein diacetate; Nrf2, nuclear factor erythroid 2-related factor 2; HO-1, hemeoxygenase 1; CAT, catalase; NQO1, NAD $(\mathrm{P}) \mathrm{H}$ quinone oxidoreductase.

\section{Acknowledgements}

This study was supported by the National Natural Science Foundation of China (№ 81373478; 81302835; 81370750), the Jiangsu Province Science Foundation for Youths (No BK20130951) and the Jiangsu Province "Qing-Lan project" for excellent young backbone teachers.

\section{Author contribution}

Bo MA and Qi ZHANG designed the research; Yu WANG, Suhan GUO, Li-sha YU, Jian-wei ZHU, Ang ZHAO and Yan-fen ZHOU performed the research; Xue-jun SHANG analyzed the data; and Guo-hua AN and Bo MA wrote the paper.

\section{Supplementary information}

Supplementary information is available at the website of Acta Pharmacologica Sinica. 


\section{References}

1 Lu Y, Bao X, Sun T, Xu J, Zheng W, Shen P. Triptolide attenuate the oxidative stress induced by LPS/D-GaIN in mice. J Cell Biochem 2012; 113: 1022-33.

2 Fu Q, Jiang ZZ, Zhang LY. Impairment of triptolide on liver mitochondria in isolated liver mitochondria and HL7702 cell line. Chin J Integr Med 2013; 19: 683-8.

3 Kupchan SM, Court WA, Dailey RG Jr, Gilmore CJ, Bryan RF. Triptolide and tripdiolide, novel antileukemic diterpenoid triepoxides from Tripterygium wilfordii. J Am Chem Soc 1972; 94: 7194-5.

4 Wang B, Ma L, Tao X, Lipsky PE. Triptolide, an active component of the Chinese herbal remedy Tripterygium wilfordii Hook F, inhibits production of nitric oxide by decreasing inducible nitric oxide synthase gene transcription. Arthritis Rheum 2004; 50: 2995-303.

5 Lin N, Sato T, Ito A. Triptolide, a novel diterpenoid triepoxide from Tripterygium wilfordii Hook. f., suppresses the production and gene expression of pro-matrix metalloproteinases 1 and 3 and augments those of tissue inhibitors of metalloproteinases 1 and 2 in human synovial fibroblasts. Arthritis Rheum 2001; 44: 2193-200.

6 Wan CK, Wang C, Cheung HY, Yang M, Fong WF. Triptolide induces $\mathrm{Bcl}-2$ cleavage and mitochondria dependent apoptosis in p53deficient HL-60 cells. Cancer Lett 2006; 241: 31-41.

7 Li H, Takai N, Yuge A, Furukawa Y, Tsuno A, Tsukamoto Y, et al. Novel target genes responsive to the anti-growth activity of triptolide in endometrial and ovarian cancer cells. Cancer Lett 2010; 297: 198206.

8 Pan J. RNA polymerase-an important molecular target of triptolide in cancer cells. Cancer Lett 2010; 292: 149-52.

9 Li CJ, Chu CY, Huang LH, Wang MH, Sheu LF, Yeh Jl, et al. Synergistic anticancer activity of triptolide combined with cisplatin enhances apoptosis in gastric cancer in vitro and in vivo. Cancer Lett 2012; 319: 203-13.

10 Kim HJ, Ravichandran K, Ozkok A, Wang Q, He Z, Jani A, et al. The water-soluble triptolide derivative $P G 490-88$ protects against cisplatin-induced acute kidney injury. J Pharmacol Exp Ther 2014; 349: 518-25.

11 Li J, Jin J, Li M, Guan C, Wang W, Zhu S, et al. Role of Nrf2 in protection against triptolide-induced toxicity in rat kidney cells. Toxicol Lett 2012; 213: 194-202.

12 Li J, Shen F, Guan C, Wang W, Sun X, Fu X, et al. Activation of Nrf2 protects against triptolide-induced hepatotoxicity. PLoS One 2014; 9:e100685.

13 Wang X, Jiang Z, Xing M, Fu J, Su Y, Sun L, et al. Interleukin-17 mediates triptolide-induced liver injury in mice. Food Chem Toxicol 2014; 71: 33-41.

14 Guan CW, Jin J, Li J, Zhao ZX, Huang ZY. Tanshinone IIA protects against triptolide-induced liver injury via Nrf2/ARE activation. Yao Xue Xue Bao 2013; 48: 1397-402.

15 Dhar P, Singla N. Histomorphological and biochemical changes induced by triptolide treatment in male lesser bandicoot rat, Bandicota bengalensis. Pestic Biochem Physiol 2014; 116: 49-55.

$16 \mathrm{Ni} \mathrm{B}$, Jiang Z, Huang X, Xu F, Zhang R, Zhang Z, et al. Male reproductive toxicity and toxicokinetics of triptolide in rats. Arzneimittelforschung 2008; 58: 673-80.

17 Singla N, Kaur G, Babbar BK, Sandhu BS. Potential of triptolide in reproductive management of the house rat, Rattus rattus. Integr Zool 2013; 8: 260-76.

18 Xue M, Zhao Y, Li XJ, Jiang ZZ, Zhang L, Liu SH, et al. Comparison of toxicokinetic and tissue distribution of triptolide-loaded solid lipid nanoparticles vs free triptolide in rats. Eur J Pharm Sci 2012; 47: 713-7.

19 Ma B, Qi H, Li J, Xu H, Chi B, Zhu J, et al. Triptolide disrupts fatty acids and peroxisome proliferator-activated receptor (PPAR) levels in male mice testes followed by testicular injury: a GC-MS based metabolomics study. Toxicology 2015; 336: 84-95.

20 Griswold MD. The central role of Sertoli cells in spermatogenesis. Semin Cell Dev Biol 1998; 9: 411-6.

21 Liu Q, Chen T, Chen H, Zhang M, Li N, Lu Z, et al. Triptolide (PG-490) induces apoptosis of dendritic cells through sequential p38 MAP kinase phosphorylation and caspase 3 activation. Biochem Biophys Res Commun 2004; 319: 980-6.

22 Xu P, Wang H, Li Z, Yang Z. Triptolide attenuated injury via inhibiting oxidative stress in amyloid-Beta-treated differentiated PC12 cells. Life Sci 2016; 145: 19-26.

23 Zhang J, Liu L, Mu X, Jiang Z, Zhang L. Effect of triptolide on estradiol release from cultured rat granulosa cells. Endocr J 2012; 59: 473-81.

24 Yao J, Jiang Z, Duan W, Huang J, Zhang L, Hu L, et al. Involvement of mitochondrial pathway in triptolide-induced cytotoxicity in human normal liver L-02 cells. Biol Pharm Bull 2008; 31: 592-7.

25 Sun L, Li H, Huang X, Wang T, Zhang S, Yang J, et al. Triptolide alters barrier function in renal proximal tubular cells in rats. Toxicol Lett 2013; 223: 96-102.

26 Yang F, Zhuo L, Ananda S, Sun T, Li S, Liu L. Role of reactive oxygen species in triptolide-induced apoptosis of renal tubular cells and renal injury in rats. J Huazhong Univ Sci Technol Med Sci 2011; 31: 33541.

27 Zhang X, Lui WY. Transforming growth factor- $\beta 3$ regulates cell junction restructuring via MAPK-mediated mRNA destabilization and Smaddependent protein degradation of junctional adhesion molecule $B$ (JAM-B). Biochim Biophys Acta 2015; 1849: 601-11.

28 Thuillier R, Manku G, Wang Y, Culty M. Changes in MAPK pathway in neonatal and adult testis following fetal estrogen exposure and effects on rat testicular cells. Microsc Res Tech 2009; 72: 773-86.

29 Shi Y, Song Y, Wang Y, Wang Y, Liang X, Hu Y, et al. beta-Benzene hexachloride induces apoptosis of rat Sertoli cells through generation of reactive oxygen species and activation of JNKs and FasL. Environ Toxicol 2011; 26: 124-35.

30 Song Y, Shi Y, Yu H, Hu Y, Wang Y, Yang K. p,p'-Dichlorodiphenoxydi chloroethylene induced apoptosis of Sertoli cells through oxidative stress-mediated p38 MAPK and mitochondrial pathway. Toxicol Lett 2011; 202: 55-60.

31 Qi S, Fu W, Wang C, Liu C, Quan C, Kourouma A, et al. BPA-induced apoptosis of rat Sertoli cells through Fas/FasL and JNKs/p38 MAPK pathways. Reprod Toxicol 2014; 50: 108-16.

32 Wajda A, Łapczuk J, Grabowska M, Słojewski M, Laszczyńska M, Urasińska E, et al. Nuclear factor E2-related factor-2 (Nrf2) expression and regulation in male reproductive tract. Pharmacol Rep 2016; 68: 101-8.

33 Liu W, Yang B, Wu L, Zou W, Pan X, Zou T, et al. Involvement of NRF2 in perfluorooctanoic acid-induced testicular damage in male mice. Biol Reprod 2015; 93: 41.

34 Tan BJ, Chiu GN. Role of oxidative stress, endoplasmic reticulum stress and ERK activation in triptolide-induced apoptosis. Int J Oncol 2013; 42: 1605-12.

35 Wu Y, Cui J, Bao X, Chan S, Young DO, Liu D, et al. Triptolide attenuates oxidative stress, NF-kappaB activation and multiple cytokine gene expression in murine peritoneal macrophage. Int J Mol Med 2006; 17: 141-50.

36 Wu PP, Liu KC, Huang WW, Ma CY, Lin H, Yang JS, et al. Triptolide induces apoptosis in human adrenal cancer $\mathrm{NCl}-\mathrm{H} 295$ cells through a mitochondrial-dependent pathway. Oncol Rep 2011; 25: 551-7.

$37 \mathrm{Hu} \mathrm{J,} \mathrm{Yu} \mathrm{Q,} \mathrm{Zhao} \mathrm{F,} \mathrm{Ji} \mathrm{J,} \mathrm{Jiang} \mathrm{Z,} \mathrm{Chen} \mathrm{X,} \mathrm{et} \mathrm{al.} \mathrm{Protection} \mathrm{of} \mathrm{Quercetin}$ 
against triptolide-induced apoptosis by suppressing oxidative stress in rat Leydig cells. Chem Biol Interact 2015; 240: 38-46.

38 Haberzettl P, Hill BG. Oxidized lipids activate autophagy in a JNKdependent manner by stimulating the endoplasmic reticulum stress response. Redox Biol 2013; 1: 56-64.

39 Zhang Y, Chen F. Reactive oxygen species (ROS), troublemakers between nuclear factor-kappaB (NF-kappaB) and c-Jun $\mathrm{NH}_{2}$-terminal kinase (JNK). Cancer Res 2004; 64: 1902-5.

40 Aoki H, Kang PM, Hampe J, Yoshimura K, Noma T, Matsuzaki M, et al. Direct activation of mitochondrial apoptosis machinery by c-Jun $\mathrm{N}$-terminal kinase in adult cardiac myocytes. J Biol Chem 2002; 277 : 10244-50.

41 Schroeter H, Boyd CS, Ahmed R, Spencer JP, Duncan RF, Rice-Evans
C, et al. c-Jun N-terminal kinase (JNK)-mediated modulation of brain mitochondria function: new target proteins for JNK signalling in mitochondrion-dependent apoptosis. Biochem J 2003; 372: 359-69.

42 Lei K, Nimnual A, Zong WX, Kennedy NJ, Flavell RA, Thompson CB, et al. The Bax subfamily of $\mathrm{Bcl}-2$-related proteins is essential for apoptotic signal transduction by $\mathrm{c}$-Jun $\mathrm{NH}_{2}$-terminal kinase. Mol Cell Biol 2002; 22: 4929-42.

43 Wei A, Zhou D, Xiong C, Cai Y, Ruan J. A novel non-aromatic B-ring flavonoid: isolation, structure elucidation and its induction of apoptosis in human colon HT-29 tumor cell via the reactive oxygen species-mitochondrial dysfunction and MAPK activation. Food Chem Toxicol 2011; 49: 2445-52. 